\title{
Fas ligand promotes an inducible TLR-dependent model of cutaneous lupus-like inflammation
}

\author{
Purvi Mande, ${ }^{1}$ Bahar Zirak, ${ }^{2}$ Wei-Che Ko, ${ }^{3}$ Keyon Taravati, ${ }^{2}$ Karen L. Bride, ${ }^{4}$ Tia Y. Brodeur, ${ }^{1}$ April Deng, ${ }^{3}$ Karen Dresser, ${ }^{3}$ \\ Zhaozhao Jiang, ${ }^{1}$ Rachel Ettinger, ${ }^{5}$ Katherine A. Fitzgerald, ${ }^{1}$ Michael D. Rosenblum, ${ }^{2}$ John E. Harris, ${ }^{3}$ \\ and Ann Marshak-Rothstein ${ }^{1,4}$ \\ 'Department of Medicine, University of Massachusetts School of Medicine, Worcester, Massachusetts, USA. ²Department of Dermatology, UCSF, San Francisco, California, USA. ${ }^{3}$ Department of Dermatology, \\ University of Massachusetts School of Medicine, Worcester, Massachusetts, USA. "Department of Microbiology, Boston University School of Medicine, Boston, Massachusetts, USA. \\ ${ }^{5}$ Respiratory, Autoimmunity, and Inflammation Department, Medlmmune, Gaithersburg, Maryland, USA.
}

\begin{abstract}
Toll-like receptors TLR7 and TLR9 are both implicated in the activation of autoreactive B cells and other cell types associated with systemic lupus erythematosus (SLE) pathogenesis. However, TIr9-/- autoimmune-prone strains paradoxically develop more severe disease. We have now leveraged the negative regulatory role of TLR9 to develop an inducible rapid-onset murine model of systemic autoimmunity that depends on T cell detection of a membrane-bound OVA fusion protein expressed by MHC class II' cells, expression of TLR7, expression of the type I IFN receptor, and loss of expression of TLR9. These mice are distinguished by a high frequency of OVA-specific Tbet $^{+}$, IFN- $\gamma^{+}$, and FasL-expressing Th1 cells as well as autoantibodyproducing B cells. Unexpectedly, contrary to what occurs in most models of SLE, they also developed skin lesions that are very similar to those of human cutaneous lupus erythematosus (CLE) as far as clinical appearance, histological changes, and gene expression. FasL was a key effector mechanism in the skin, as the transfer of FasL-deficient D0119ld $\mathrm{T}$ cells completely failed to elicit overt skin lesions. FasL was also upregulated in human CLE biopsies. Overall, our model provides a relevant system for exploring the pathophysiology of CLE as well as the negative regulatory role of TLR9.
\end{abstract}

\section{Introduction}

Cutaneous lupus erythematosus (CLE) refers to dermatologic features of systemic lupus erythematosus (SLE) that may or may not be associated with more systemic manifestations, such as arthritis, nephritis, or neurological disorders $(1,2)$. The most common cutaneous lesions in CLE patients are characterized histologically as interface dermatitis associated with epidermal basal-cell damage, apoptosis of keratinocytes (KCs), and a variable subepithelial inflammatory infiltrate in the skin that includes cytotoxic Th1 cells and plasmacytoid dendritic cells (pDCs) (2-5). Common pathophysiological mechanisms, including proinflammtory type 1 IFN, are thought to promote CLE and other clinical manifestations of SLE (7). Therefore, a better understanding of the genetic, environmental, and immunoregulatory factors that drive CLE is likely to provide important insights for the treatment of both CLE and SLE. However, remarkably little is known about the mechanisms that distinguish the different subcategories of CLE or cause CLE patients to progress to SLE, in part due to the lack of appropriate animal models. MRL/lpr is the most commonly studied strain in this context (8), but the onset of cutaneous disease in MRL/lpr mice is highly variable, is colony dependent, lacks a number of the key features of human CLE, and takes 6 months or more to develop.

Conflict of interest: RE was an employee at Medlmmune and owns AstraZeneca stock. She is currently an employee and shareholder at VielaBio.

Submitted: October 23, 2017; Accepted: April 17, 2018.

Reference information: J Clin Invest. 2018;128(7):2966-2978.

https://doi.org/10.1172/JCI98219.
There is a general consensus that environmental factors trigger autoimmune disease in genetically predisposed individuals. These environmental triggers are often associated with tissue damage, followed by failure to effectively clear cell debris. The excessive accumulation of debris is detected by innate immune sensors and eventually leads to a break in self-tolerance. Studies from our group and others have strongly implicated nucleic acidsensing TLRs in the activation of autoreactive B cells, pDCs, and other cell types implicated in SLE pathogenesis (9-13). It follows that in all murine models of SLE evaluated to date, mice with functional defects in both TLR7 and TLR9, or simply TLR7, have markedly reduced immune activation and end-organ disease (14-23). However, quite paradoxically, TLR9 deficiency (TLR9 ${ }^{\mathrm{KO}}$ ) alone invariably leads to exacerbated clinical manifestations in these same models, despite the fact that functional TLR9 (TLR9WT) is required for the production of dsDNA/chromatin-reactive autoAbs (15, 24-27). Exactly how the loss of TLR9 can have such a profound impact on disease progression is unclear, especially since the resulting autoimmune disorders may reflect a variety of tissuespecific effector mechanisms.

We have now leveraged the hyperactivity of TLR9-deficient mice to develop a T cell-dependent model of systemic autoimmunity. Clinical manifestations of this model depend on the adoptive transfer of OVA-specific DO11 T cells into sublethally irradiated TLR9 ${ }^{\mathrm{KO}}$ mice that express a Tg-encoded OVA fusion protein on MHC class $\mathrm{II}^{+}$cells. Remarkably, these mice develop a systemic autoimmune disease with severe cutaneous manifestations, but only if the recipient is TLR7 sufficient and the DO11 T cells are capable of expressing FasL. Gene expression profiling revealed 
A

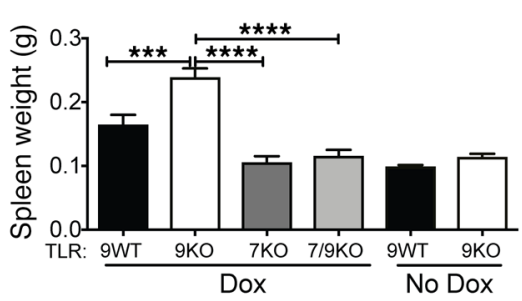

C

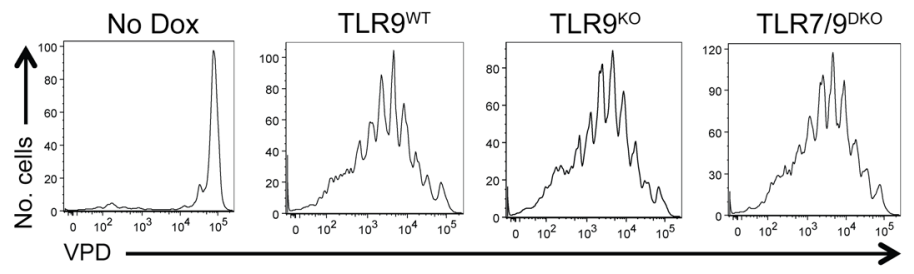

D

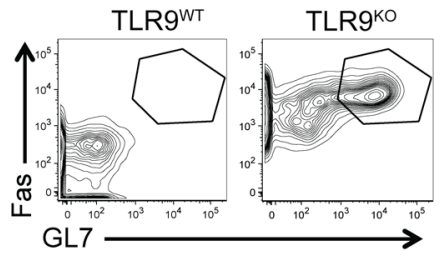

E
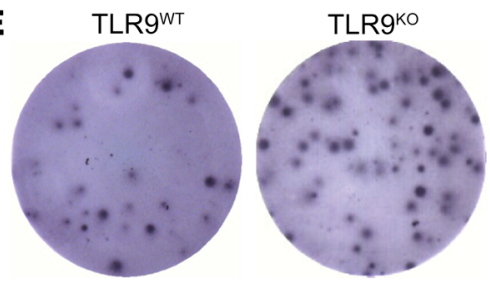

F TLR9 ${ }^{\text {WT }}$ Dox

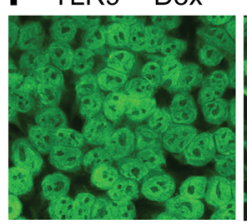

TLR9ko Dox

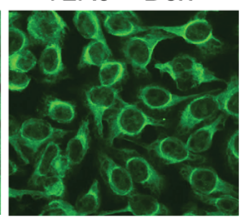

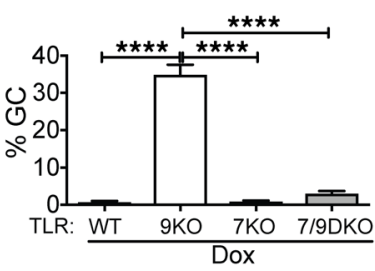
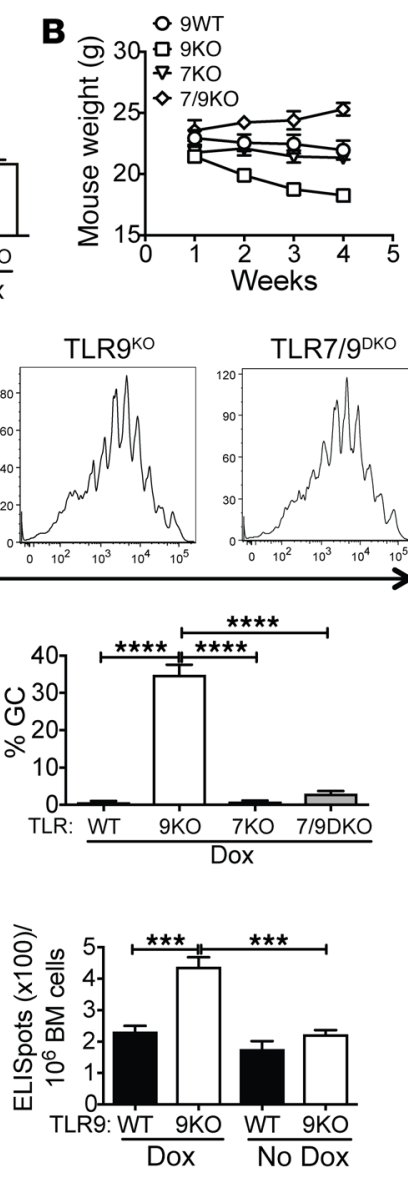

TLR7 $7^{\mathrm{KO}}$ Dox

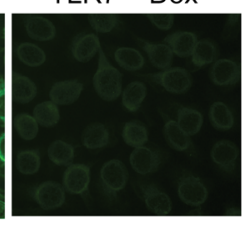

TLR9ко No Dox

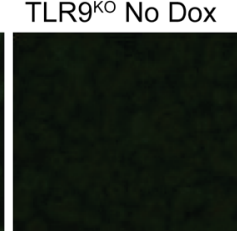

Figure 1. TLR9 deficiency promotes systemic autoimmunity and

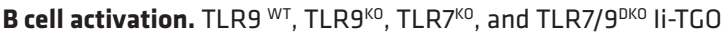
mice were or were not provided with Dox chow and were sublethally irradiated (400R) and injected i.v. with D011 T cells. (A and B) Spleen weight (g) at 4 weeks and total body weight $(\mathrm{g})$ at weekly time points following injection. (c) Proliferation of VPD-labeled naive D011 T cells in sdLNs 5 days after T cell injection ( $n=5$ per group). (D) B220+ cells from the sdLNs stained for GC markers Fas and GL7. (E) Plasma cells in the bone marrow measured by ELISpot assay at 4 weeks after T cell injection ( $n=6$ per group). (F) Autoantibodies detected by HEp2 staining. Original magnification, $\times 200$. Images were captured at $\times 2$ magnification using an ImmunoSpot plate reader (CTL), and a representative well image is shown in the figure. Data are shown as mean \pm SEM and are representative of 5 independent experiments with $n=20$ mice per group (A, B, D, and $\mathbf{F}) .{ }^{* *} P<0.001 ;{ }^{* * *} P<0.0001,1$-way ANOVA with Šidák's multiple-comparison test.

As expected, CFSE-labeled OVA-specific DO11 Tg T cells only divide when injected into double-transgenic Ii-TGO mice simultaneously administered Dox. Expression of either transgene alone was not sufficient to induce division (Supplemental Figure 1, A and B; supplemental material available online with this article; https://doi.org/10.1172/ JCI98219DS1). Nevertheless, Ii-TGO mice injected with naive DO11 T cells showed no evidence of sustained autoimmunity. However, when Dox-administered Ii-TGO mice were sublethally irradiated (Dox/400R) and then injected with preactivated $\mathrm{T}$ cells, they developed modest splenomegaly and produced autoantibodies (Supplemental Figure 1, C and D). Antinuclear antibodies (ANAs) were detected by 3 to 4 weeks after $\mathrm{T}$ cell injection by immunofluorescent staining of HEp-2 cells. These sera showed a predominantly homogeneous nuclear-staining pattern, consistent with a role for TLR9. Beyond splenomegaly and ANA production, these mice did not exhibit further clinical manifestations of autoimmune disease.

TLR9 constrains self-reactivity in Ii-TGO mice. To determine whether TLR9 deficiency would lead to a

that FasL is also upregulated in the skin of CLE patients compared with healthy controls, while FasL is not upregulated in the skin of psoriasis patients. Thus, we have developed a rapidly inducible model of cutaneous lupus that allows us to explore the negative regulatory role of TLR9 as well as the pathophysiology of CLE.

\section{Results}

$T$ cell detection of an OVA fusion protein expressed on $\mathrm{MHC}$ class $\mathrm{II}^{+}$ cells induces autoantibody production. Mice expressing a membrane-bound OVA Tg have been described previously (28-30). This fusion protein, referred to as TGO, incorporates OVA $^{1122-1595}$ fused to the transmembrane and cytoplasmic domains of the transferrin receptor, and therefore TGO efficiently traffics to endocytic compartments. The promoter is a Tet-regulated element (TRE), so gene transcription depends on the combination of a reverse transactivator (rtTA) and doxycycline (Dox). We crossed the TGO mice to a $\mathrm{Tg}$ line expressing an invariant chain-driven (Ii-driven) rtTA (Ii-rtTA) (31), resulting in TGO expression on MHC class $\mathrm{II}^{+}$cells following Dox administration. These double-Tg Ii-TGO mice were then backcrossed to BALB/c for over 10 generations. more severe disease outcome in this model, we intercrossed IiTGO and BALB/c TLR9-deficient (TLR9 ${ }^{\mathrm{KO}}$ ) mice. Remarkably, DO11-injected TLR9 ${ }^{\mathrm{KO}}$ Ii-TGO mice developed more extensive splenomegaly than TLR9-sufficient (TLR9 ${ }^{\mathrm{WT}}$ ) Ii-TGO mice (Figure 1A) and also began to lose weight (Figure 1B). Comparably treated TLR7-deficient $\left(\mathrm{TLR}^{\mathrm{KO}}\right)$ or TLR7/9 double-deficient (TLR7/9 ${ }^{\mathrm{DKO}}$ ) Ii-TGO recipient mice showed no evidence of splenomegaly or weight loss. The inability of DO11 $\mathrm{T}$ cells to trigger an autoimmune response in the TLR $7^{\mathrm{KO}}$ and TLR $7 / 9^{\mathrm{DKO}}$ mice was not due to the inability of $\mathrm{T}$ cells to engraft or the recipient cells to express antigen, as fluorochrome-labeled (VPD450, Violet Proliferation Dye) $\mathrm{T}$ cells divided comparably in all genotypes (Figure 1C). Recipient mice that were not provided with Dox also failed to show any signs of autoimmunity. Therefore, the enhanced autoimmune response detected in the TLR9 ${ }^{\mathrm{KO}}$ Ii-TGO recipients depended on Dox-induced expression of TGO and the natural expression of TLR7 as well as loss of TLR9.

TLR9 deficiency further promotes B cell activation. TLR9 ${ }^{\mathrm{KO}}$ IiTGO recipients mounted more vigorous B cell responses than TLR9 ${ }^{\text {WT }}$ Ii-TGO recipients, as shown by a higher proportion of 

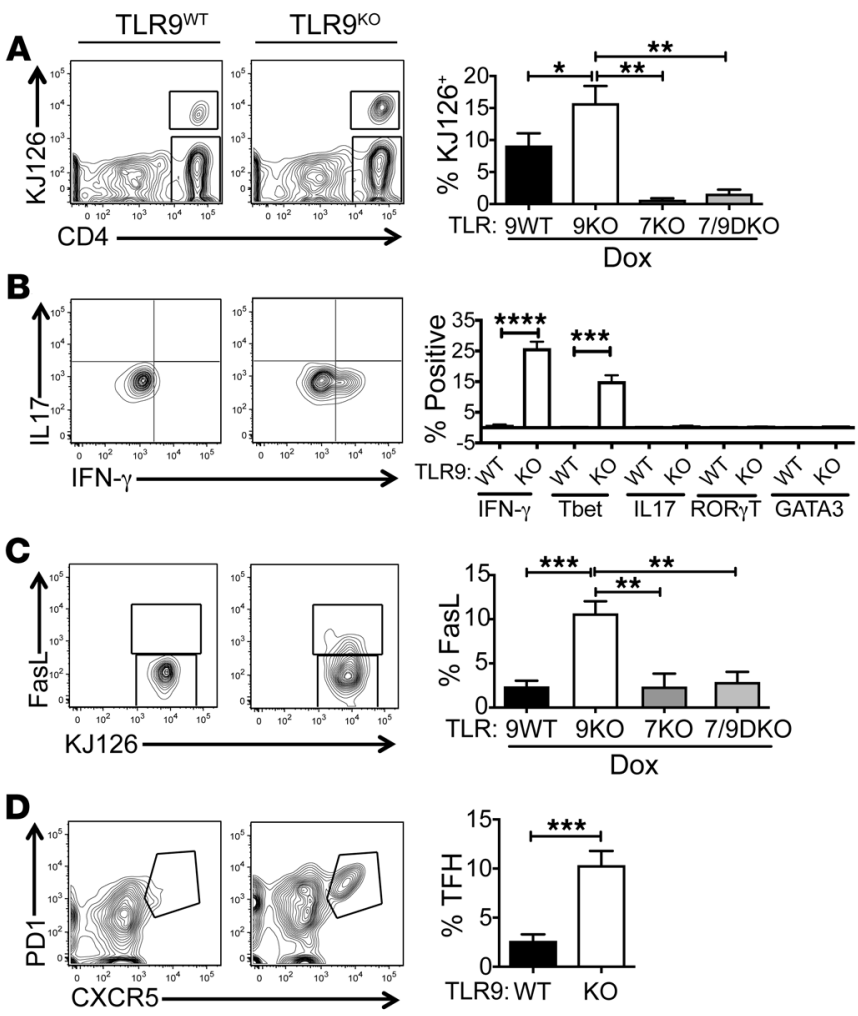

Figure 2. TLR9-deficiency promotes development of Th1 and TFH cells. sdLN suspensions from D011-injected TLR9' ${ }^{\mathrm{WT}}$, TLR9 ${ }^{\mathrm{Ko}}, \mathrm{TLR}^{\mathrm{KO}}$, and TLR7/9סK |i-TCO mice at 4 weeks after T cell injection were analyzed for (A) percentages of D011 (KJ126+) T cells in the CD4+ gate, (B) percentages of cytokine-producing cells in the $\mathrm{KJ}^{126^{+}}$gate, (C) percentages of FasLexpressing cells in the $\mathrm{KJ} 126^{+}$gate, and (D) percentages of TFH cells in the $\mathrm{KJ} 126^{+}$gate. Data are shown as mean $\pm \mathrm{SEM}$ and are representative of 5 independent experiments with $n=15$ mice per group. ${ }^{*} P<0.05$; ${ }^{* *} P<$ $0.01 ;{ }^{* *} P<0.001 ;{ }^{* * *} P<0.0001,1$-way ANOVA with Šidák's multiplecomparison test (A-C) and 2-tailed Student's $t$ test (D).

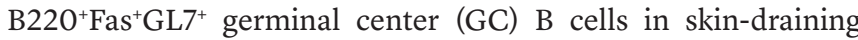
LNs (sdLNs) and spleen by 4 weeks after $\mathrm{T}$ cell injection (Figure 1D and Supplemental Figure 2A). They also had more ELISpot ${ }^{+}$ plasma cells in the BM and spleen when compared with TLR9 ${ }^{\text {WT }}$ recipients (Figure 1E and Supplemental Figure 2B). As predicted from previous studies $(20,32)$, TLR expression modulated autoantibody specificity, as shown by ANA staining patterns on HEp2 cells; sera from the TLR9 ${ }^{\mathrm{WT}}$ mice showed a predominantly homogeneous nuclear-staining pattern, while the TLR ${ }^{\mathrm{KO}}$ sera routinely showed a cytoplasmic staining pattern (Figure 1F). This is an ANA pattern associated with SLE (AC-19; International Consensus on ANA Patterns, www.anapatterns.org), and we have seen this pattern frequently in mice with predominantly TLR7-driven disease. $\mathrm{GC}^{+} \mathrm{B}$ cells were not detected in TLR7 ${ }^{\mathrm{KO}}$ or TLR7/9 $9^{\mathrm{DKO}}$ Ii-TGO recipients, and neither of these strains made ANAs, again pointing to a critical role for TLR7 in the development of autoimmunity.

TLR9 deficiency promotes OVA-specific T cell activation in IiTGO-expressing recipients. The impact of TLR9 deficiency on DO11 $\mathrm{T}$ cell expansion and differentiation was evaluated by flow cytometry. Both sdLNs and spleens of the TLR9 ${ }^{\mathrm{KO}}$ recipients contained a higher proportion of $\mathrm{KJ}^{2} 126^{+} \mathrm{T}$ cells than those of the
TLR9 ${ }^{\text {WT }}$ recipients, while even fewer DO11 T cells were recovered from the lymphoid tissues of comparably treated TLR $7^{\mathrm{KO}}$ or TLR7/9 ${ }^{\text {DKO }}$ Ii-TGO mice (Figure $2 \mathrm{~A}$ and Supplemental Figure $2 \mathrm{C}$ ). In addition, a high proportion of the DO11 $\mathrm{T}$ cells from the TLR9 ${ }^{\mathrm{KO}}$ Ii-TGO recipients were Tbet $^{+}$and actively producing IFN- $\gamma$, while ROR $\gamma$ T and GATA3 were not detected. In contrast, there were essentially no cytokine-producing cells in the TLR9 $^{\text {WT }}$, TLR7 ${ }^{\mathrm{KO}}$, or TLR7/9 ${ }^{\text {DKO }}$ Ii-TGO recipients (Figure $2 \mathrm{~B}$ and Supplemental Figure 2D). Consistent with their Th1 phenotype, the $\mathrm{T}$ cells in the TLR9 ${ }^{\mathrm{KO}}$ recipients also expressed significantly higher levels of FasL than any of the other groups (Figure 2C). TLR9 ${ }^{\mathrm{KO}}$ recipients also had a greater number of PD1 $1^{+} \mathrm{CXCR} 5^{+}$ $\mathrm{T}$ follicular helper (TFH) cells in the sdLNs (Figure 2D). These studies demonstrate a critical role for recipient TLR expression in the determination of $\mathrm{T}$ cell function. In the absence of TLR9, DO11 $\mathrm{T}$ cells differentiate to potent Th1-like effector cells and TFH cells through a process dependent on TLR7.

TLR9 deficiency promotes extensive immune activation in the skin. Another major difference between TLR9 ${ }^{\mathrm{WT}}$ and TLR9 ${ }^{\mathrm{KO}}$ Ii-TGO recipients was the presence of skin disease only in the TLR9 ${ }^{\mathrm{KO}}$ mice. By 4 weeks after T cell transfer, the TLR9 ${ }^{\mathrm{KO}}$ Ii-TGO recipients developed severe skin lesions over the entire torso. Histological examination revealed many of the features of human CLE: perivascular/perifollicular mononuclear infiltrate, follicular plugging, basal layer vacuolar changes, modest basement membrane thickening, dermal mucin accumulation, apoptotic cell death in the epidermis, and $\mathrm{Ab}$ deposition at the dermal/epidermal border (Figure 3, A-G, and refs. 3, 4, 7, 33-35). However, there are differences between murine lupus-like skin inflammation (LLSI) and human CLE skin. The epidermis of mouse skin is much thinner at baseline (only 2 layers) and invariably thickens in response to inflammation. Therefore, in contrast to human cutaneous lupus, which is commonly associated with epidermal atrophy, the DO11 $\rightarrow$ TLR9 ${ }^{\text {KO }}$ Ii-TGO recipients developed epidermal hyperplasia, similar to the inflammatory skin disease that occurs in SLE-prone MRL/lpr mice (36-38) or in a limited number of hypertropic discoid lupus patients (39-42). The TLR9 ${ }^{\mathrm{WT}}$, TLR7 $7^{\mathrm{KO}}$, and TLR7/9DKO Ii-TGO recipients showed no evidence of skin inflammation, demonstrating that this model of LLSI was also dependent on the loss of TLR9 and the expression of TLR7.

Cells were isolated from the shaved dorsal skin of experimental mice. FACS analysis of cells isolated from the epidermis revealed major differences between the TLR9 ${ }^{\mathrm{WT}}$ and TLR9 ${ }^{\mathrm{KO}}$ recipients (Figure 4A), consistent with more extensive immune activation in the TLR9 ${ }^{\mathrm{KO}}$ mice. Skin-infiltrating hematopoietic cells were distinguished from host stromal cells by their expression of CD45, and the TLR9 ${ }^{\mathrm{KO}}$ recipients showed more extensive infiltration of hematopoietic cells, as indicated by the percentages of $\mathrm{CD} 45^{+}$ cells in the total cell suspension (R1). The $\mathrm{CD} 45^{+}$compartment of the TLR9 ${ }^{\mathrm{KO}}$ epidermis included many more $\mathrm{CD}^{+}$cells (R3), most of which were $\mathrm{KJ}^{126^{+}} \mathrm{DO} 11 \mathrm{~T}$ cells. Moreover, the TLR9 ${ }^{\mathrm{KO}} \mathrm{CD} 11 \mathrm{c}^{+}$ cells (R4) were more activated, as shown by increased expression of both CD86 and Fas. In addition, approximately $20 \%$ of the TLR9 ${ }^{\mathrm{KO}}$ viable $\mathrm{CD}^{\text {neg }} 5^{\text {neg }} \mathrm{KCs}(\mathrm{R} 2)$, expressed MHC class II, while very few of the TLR9 ${ }^{\mathrm{WT}} \mathrm{KCs}$ were class $\mathrm{II}^{+}$. These data indicate that ongoing inflammation in the skin converted KCs to efficient TGOexpressing antigen-presenting cells (APCs). 


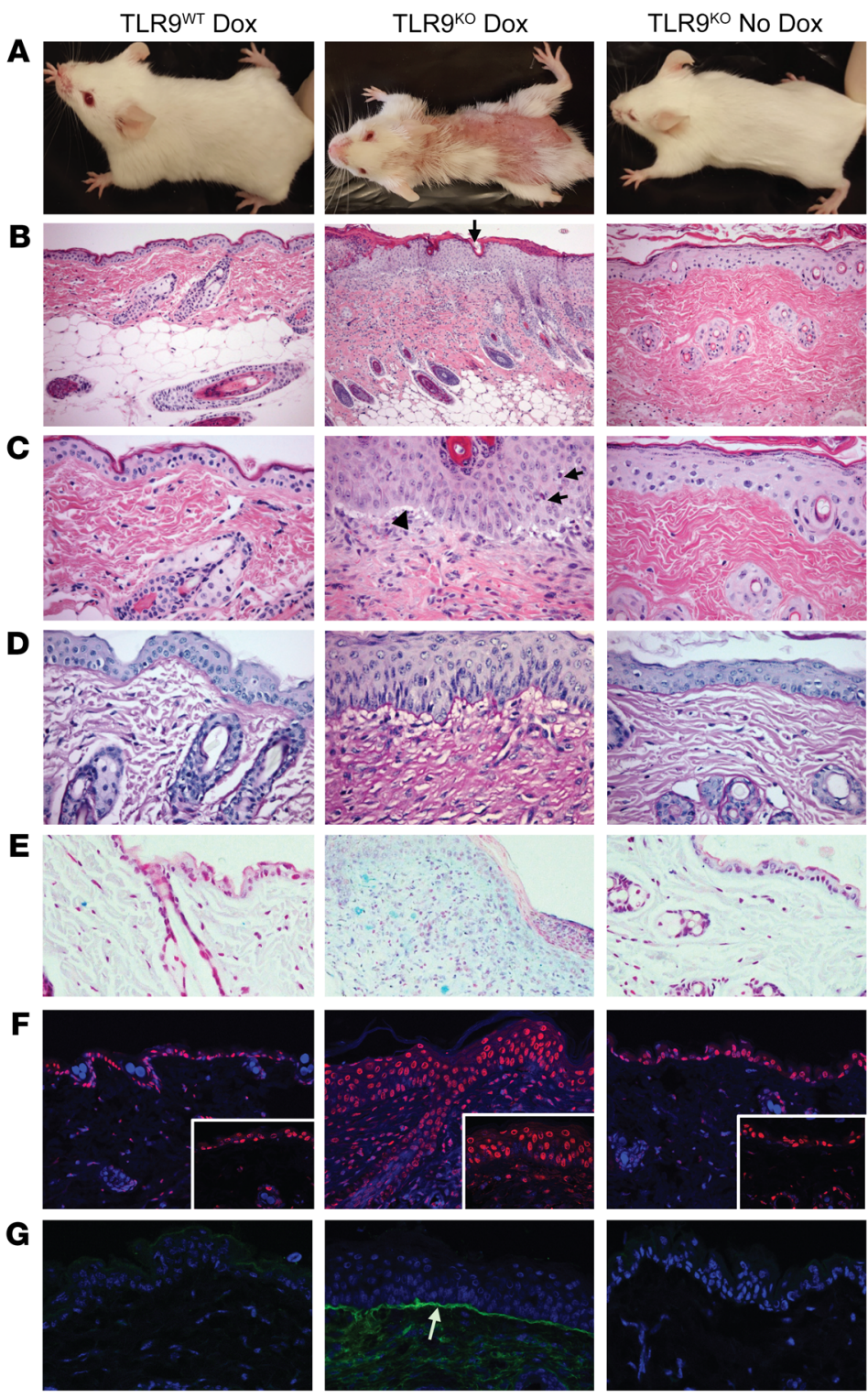

Figure 3. TLR9 deficiency results in LLSI. (A) Clinical appearance of D011-injected TLR9WT or TLR9 ${ }^{\mathrm{KO}}$ li-TCO mice, provided or not provided with Dox chow, at 4 weeks after injection. (B and C) H\&Estained skin sections showing follicular plugging (black arrow, B), perivascular and perifollicular mononuclear infiltrate, vacuolization of basal layer (black arrowhead, C), and apoptotic KCs in the epidermis (black arrows, C). Original magnification, $\times 200$ (B); ×400 (C) (D) Basement membrane thickening shown by PAS stain. Original magnification, $\times 400$. (E) Mucin deposition in the dermis detected by Alcian blue stain. Original magnification, $\times 200$. (F) TUNEL stain showing apoptotic cell death (red) counterstained with DAPI (blue). Original magnification, $\times 200 ; \times 400$ (inset). and (C) Ig deposition at basement membrane (white arrow) detected by FITC anti-IgC (green) and counterstained with DAPI (blue). Original magnification, $\times 200$. Images shown are representative of 5 mice per group from 3 independent experiments.

for FasL in cutaneous inflammation. To further explore the relevance of FasL to cutaneous disease, we compared the disease-inducing activity of FasL-deficient DO11 $1^{\text {gld }} \mathrm{T}$ cells to DO11 T cells from FasL-sufficient littermates. Remarkably, DO11 ${ }^{\text {gld }} \mathrm{T}$ cells did not elicit any overt clinical evidence of skin disease (Figure 5A). Nevertheless, the TLR9 ${ }^{\mathrm{KO}}$ mice injected with DO11gld $\mathrm{T}$ cells still developed splenomegaly and made autoantibodies (Figures 5, B and D), and FACS analysis showed evidence of immune activity in the skin (Figure $5 \mathrm{C}$ ). The DO11 ${ }^{\text {gld }} \rightarrow$ TLR9 ${ }^{\mathrm{KO}}$ mice had significantly more infiltrating inflammatory monocytes, neutrophils, and DO11 T cells compared with DO11 $\rightarrow$ TLR ${ }^{\mathrm{WT}}$ mice $(P$ $<0.05$ ) and trended toward more IFN- $\gamma$-producing DO11 T cells. However, the total number and frequency of inflammatory monocytes, granulocytes, DO11 cells, and IFN- $\gamma$ producing DO11 cells was dramatically reduced compared with that in the DO11 $\rightarrow$ TLR9 ${ }^{\mathrm{KO}}$ mice. Together, the data point to a critical role for FasL in skin inflammation.

Gene expression profiling points to FasL induction of proinflammatory cytokine and chemokine production. FasL is known to provoke both apoptosis and proinflammatory cytokine and chemokine production. To better understand

To better preserve markers compromised by the trypsin digestion used for the isolation of epidermal cells, we used a collagenase/ hyaluronidase protocol to isolate cells from unseparated epidermis and dermis (Figure 4B). Again, there were more $\mathrm{KJ} 126^{+} \mathrm{CD} 4$ cells in the TLR9 ${ }^{\mathrm{KO}}$ skin (R5), and as in the sdLNs, a significant proportion of DO11 T cells in TLR9 ${ }^{\mathrm{KO}}$, but not TLR9 ${ }^{\mathrm{WT}}$, recipients were making IFN- $\gamma(P<0.001)$. Within the $\mathrm{CD} 45^{+} \mathrm{CD} 11 \mathrm{~b}^{+}$myeloid compartment (R6), the TLR9 ${ }^{\mathrm{KO}}$ skin contained more Ly6C $\mathrm{C}^{\text {hi }}$ inflammatory monocytes (R7), Ly6 $\mathrm{C}^{\text {Int }}-\mathrm{Ly} 6 \mathrm{G}^{\text {Int }}$ cells $(\mathrm{R} 8)$, and Ly $6 \mathrm{C}^{+} \mathrm{Ly} 6 \mathrm{G}^{\text {hi }}$ granulocytes (R9). These data clearly indicate a causal relationship between infiltrating inflammatory cells and effector $\mathrm{T}$ cells and CLE-like disease and further point to a critical role for TLR9 in the negative regulation of this process. The importance of IFN- $\gamma$ to the disease process was shown by the absence of skin disease and reduction in other clinical manifestations of mice injected with IFN- $\gamma^{-1-}$ DO11 T cells (Supplemental Figure 3).

FasL is implicated in cutaneous lupus. The presence of FasLexpressing DO11 cells in the sdLNs of TLR9 ${ }^{\mathrm{KO}}$ Ii-TGO mice and the expression of Fas by KCs, T cells, and myeloid cells pointed to a role how FasL deficiency led to such a dramatic reduction in cutaneous disease, we compared gene expression patterns in the skin of DO11 $\rightarrow$ TLR9 ${ }^{\mathrm{WT}}$ Ii-TGO (group 1), DO11 $\rightarrow$ TLR9 ${ }^{\mathrm{KO}}$ Ii-TGO (group 2), and DO11 ${ }^{\text {gld }} \rightarrow$ TLR9 ${ }^{\mathrm{KO}}$ Ii-TGO (group 3) mice. RNA was isolated from total skin biopsies obtained 4 weeks after $\mathrm{T}$ cell injection, and expression levels were quantified for the 750 genes detected by the NanoString murine cancer immune code set. Normalized $\log _{2}-$ transformed values were analyzed with nSolver software 3.0, and unbiased hierarchical clustering (heatmap2 software) was used to generate the heatmap depicted in Figure 6A. A total of 156 genes were increased at least 2-fold in group 2 skin relative to group 1 skin. Unexpectedly, most of the genes highly upregulated in the group 2 skin, compared with the group 1 skin, were also upregulated in the group 3 skin (clusters II, III, V, and VII) despite the relatively normal appearance of the group 3 skin. A more limited subset of genes was only upregulated in the group 2 skin and not in the group 3 skin (clusters I, IV and VI). The fold change of the group 2 values relative to the group 1 values and the fold change of the group 3 values relative to the group 1 values were further used to classify genes as 
A
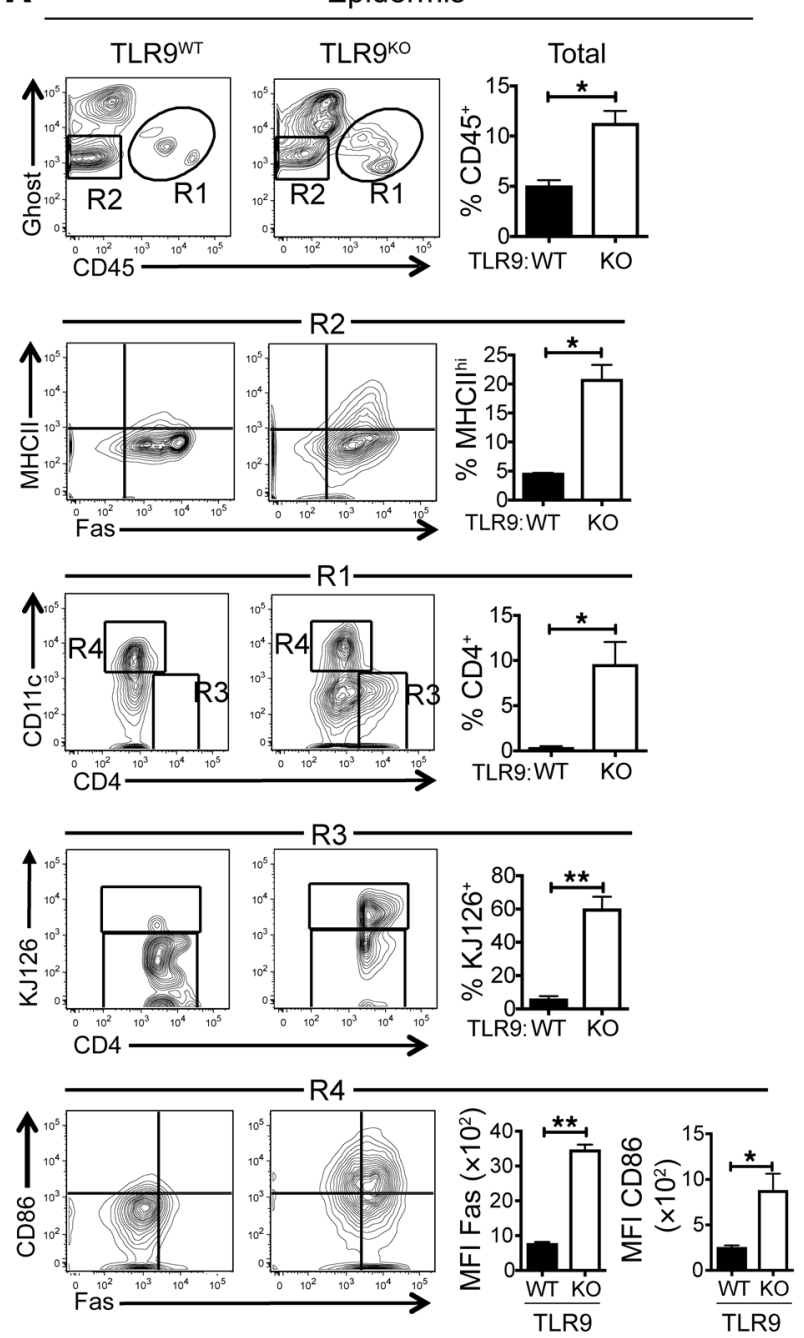

B
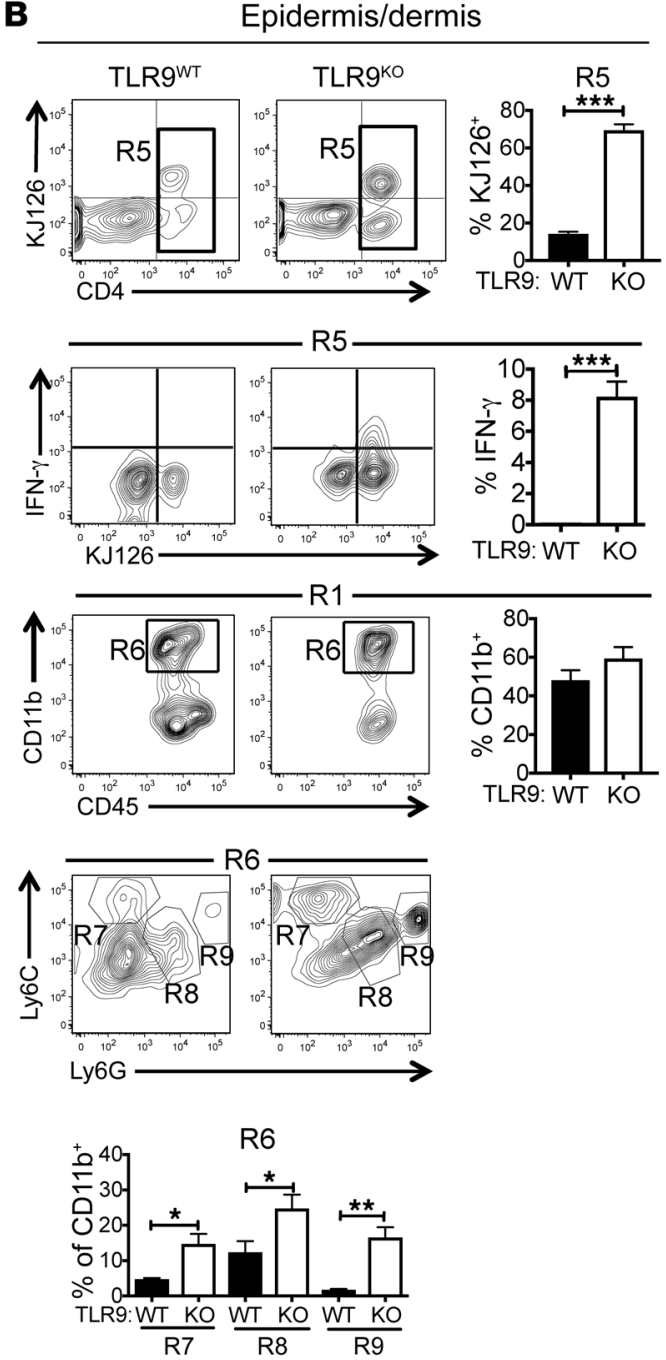

Figure 4. DO11 T cell and myeloid cell infiltration in the skin. Representative FACS plots and compiled data of cells isolated from the epidermis (A) or combined epidermis/dermis (B) of Dox/400R TLR9WT (left column) or TLRg ${ }^{\mathrm{KO}}$ (right column) recipients at 4 weeks after T cell injection. Gating summary: R1, CD45 hematopoietic cells; R2, live KCs; R3, CD45+CD4+ cells; R4, CD45+CD11c cells; R5, CD4+ cells in CD45+ gate; R6, CD11b ${ }^{+}$cells in CD45 ${ }^{+}$gate; R7, inflammatory monocytes in CD45+CD11 b+ gate; R8, Ly6C-Ly6C intermediate cells in CD45+CD11b+ gate; R9, neutrophils in CD45 $5^{+} C D 11 b^{+}$gate. Data are shown as mean \pm SEM and are representative of 3 independent experiments with $n=6$ mice per group. ${ }^{*} P<0.05$; ${ }^{* *} P<0.01$; ${ }^{* *} P<0.001$, 2-tailed Student's $t$ test.

either upregulated in both TLR9 ${ }^{\mathrm{KO}}$ recipient groups (Figure 6B) or preferentially upregulated in group 2 skin compared with group 3 skin (Figure 6C). Genes highly upregulated in both strains included many interferon-stimulated genes (ISGs), such as OAS2, IFITM1, IFI44, STAT1, CXCL9, CXCL10, and CXCL11. Genes preferentially upregulated in the group 2 skin included myeloid and neutrophil chemokines, such as IL-1ß, IL-6, CXCL2 (MIP-2), CXCL1 (KC), CCL3 (MIP-1 $\alpha$ ), and CCL4 (MIP-1 1 ), genes previously shown to be induced by FasL activation of peritoneal macrophages and other cell types $(43,44)$. A small number of genes were also lower in the group 2 skin than the other 2 groups. These included CD207 (Langerin, indicated by a black arrow in cluster III, Figure 6A). The decrease in Langerin transcripts indicates the reduced presence of Langerhans cells in the skin. Together, the data demonstrate that a remarkably small number of FasL-induced proinflammatory chemokines and cytokines are needed to recruit the effector cells that mediate actual skin lesions.
Importance of type I IFN to clinical manifestation of disease. The strong IFN signature apparent in the skin could reflect the activity of IFN- $\gamma$ and/or elevated levels of type I IFNs. The exacerbated disease of TLR9-deficient MRL/lpr mice was previously found to depend on type I IFNs (45). To determine the role of type I IFN in our model, TLR9 ${ }^{\mathrm{KO}}$ Ii-TGO mice were injected biweekly with a blocking Ab specific for the type I IFN receptor $\alpha$-chain (IFN-aR) $(46,47)$ or with an isotype control, starting from day-1 relative to $T$ cell transfer until the mice were euthanized. Ab treatment dramatically reduced clinical manifestations of disease when compared with that of littermates treated with an isotype control (Figure 7, A and B). Although the IFN-aR-treated TLR9 ${ }^{\mathrm{KO}}$ mice still developed splenomegaly (Figure 7C), there were major differences in the skin-infiltrating cells, as shown by FACS analysis, including significantly fewer $\mathrm{CD} 45^{+}$hematopoietic cells, DO11 T cells, and pDCs in the skin of the IFN-aR-treated mice compared with isotype control TLR9 ${ }^{\mathrm{KO}}$ mice (Figure 7, D and E). pDCs are a major source of 
A
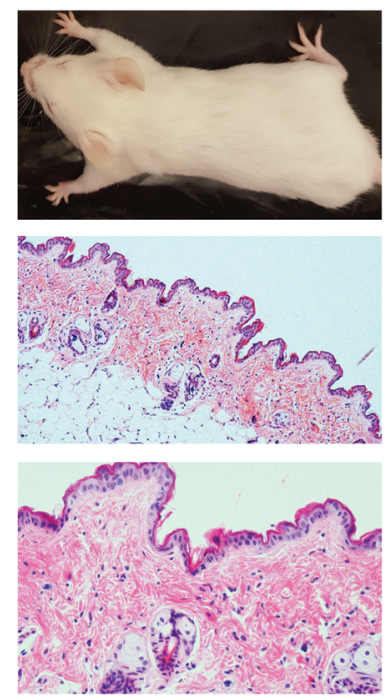

B

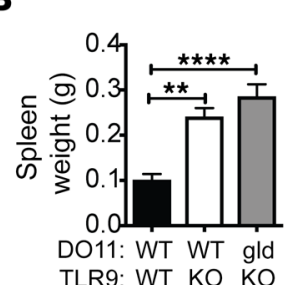

C

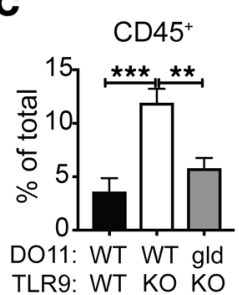

DO11 $\rightarrow$ TLR9 ${ }^{\text {Ko }}$
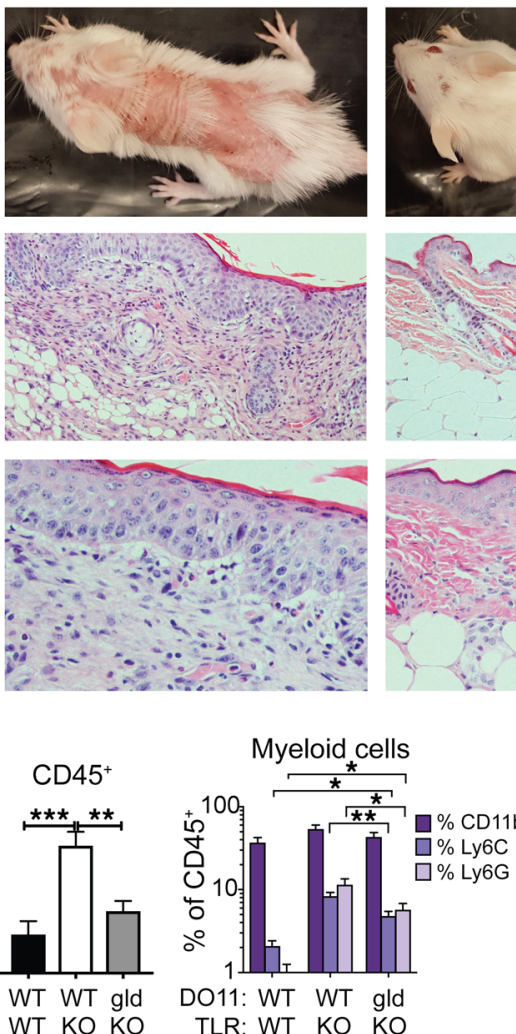

TLR: WT KO KO
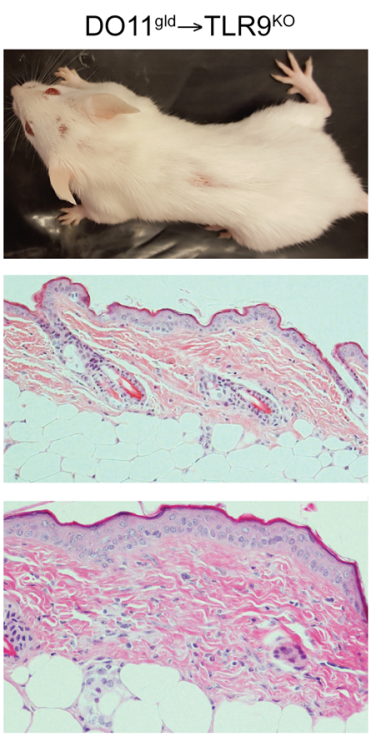

Figure 5. FasL-deficient D011 T cells fail to induce skin lesions. D011 or D0119ld T cell-injected Dox/400R TLR9 ${ }^{W T}$ or TLR9 ${ }^{\mathrm{KO}}$ liTCO recipients were evaluated 4 weeks after injection. (A) Clinical appearance (upper row) and skin histology by H\&E staining. Origina magnification, $\times 100$ (middle row); $\times 200$ magnification (bottom row). Representative images from $n=5$ mice per group. (B) Spleen weights (C) Skin-infiltrating cells, percentages of $\mathrm{CD} 45^{+}$cells in total skin cell suspension (left); percentages of CD11b $^{+}$myeloid cells, CD11b+Ly6C ${ }^{+}$ inflammatory monocytes, and CD11b+Ly6C+ ${ }^{+}$neutrophils (center); and percentages of $\mathrm{CD} 4^{+}$, CD4 ${ }^{+} \mathrm{KJ} 126^{+}$, and $\mathrm{KJ} 126^{+} \mathrm{IFN}-\gamma^{+} \mathrm{T}$ cells (right) within the $\mathrm{CD}^{4} 5^{+}$gate. (D) Autoantibodies detected by HEp2 staining and ANA score. Original magnification, $\times 200$. Data are shown as mean \pm SEM and are representative of 2 independent experiments with $n=8$ mice per group. ${ }^{*} P<0.01$; ${ }^{* *} P<0.01$; ${ }^{* *} P$ $<0.001 ;{ }^{* * * *} P<0.0001$, 1-way ANOVA and 2-way ANOVA with Šidák's multiple-comparison test.
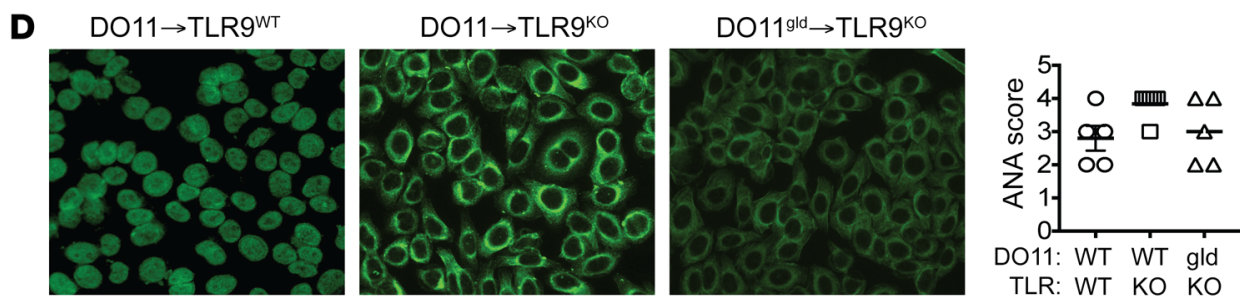

type I IFN in both SLE-prone mice and lupus patients $(48,49)$ and migrate to the skin in patients with CLE (50). Together, the data demonstrate a key role for type I IFN in this model.

Relevance to human CLE. To further explore the relevance of the DO11Ii-TGO mice to human disease, we compared gene expression profiles of lesional skin obtained from subjects with CLE or psoriasis to that of healthy controls. Clinical biopsies from the skin of patients were identified and confirmed histologically to be consistent with a diagnosis of CLE or psoriasis; the patients' charts were reviewed to determine the lupus subtype by clinical presentation, either acute cutaneous lupus (ACLE), subacute cutaneous lupus (SCLE), or chronic cutaneous lupus (CCLE). RNA isolated from these samples was analyzed using Illumina DASL gene array, as described previously (51), and further analyzed with a NanoString human cancer immune code set. Gene expression within human lupus lesional skin revealed key parallels to our murine model. Remarkably, FasL expression was significantly increased in all SCLE and CCLE subjects (trending toward significance in ACLE subjects), but not in psoriasis subjects, verifying that its expression is specific for lupus rather than all inflammatory skin diseases (Figure 8A). Gene expression data from the human
NanoString analysis was compared with the murine NanoString data by using genes that were more than 2.5 -fold upregulated in the group 2 mice compared with the group 1 mice and genes that were more than 2-fold upregulated in CCLE subjects compared with healthy controls. A number of highly upregulated genes in the murine code set were not present in the human code set and vice versa and hence were excluded from the analyses. However, many of the ISGs and chemokines highly upregulated in the murine array were also upregulated in the human lupus lesions and, to a lesser extent, in the psoriasis lesions (Figure 8B), further supporting the relevance of our model to human CLE.

\section{Discussion}

Failure to appropriately clear cell debris is a common feature of lupus and other related disorders. Nucleic acid sensors, such as the endosomal TLRs, then respond to excessive levels of DNA and/or RNA by producing type I IFNs and proinflammatory cytokines that promote tissue-specific inflammatory responses. For reasons that remain unresolved, TLR9-deficient SLE-prone mice invariably develop more severe clinical manifestations than their TLR9-sufficient counterparts through mechanisms that depend 
A
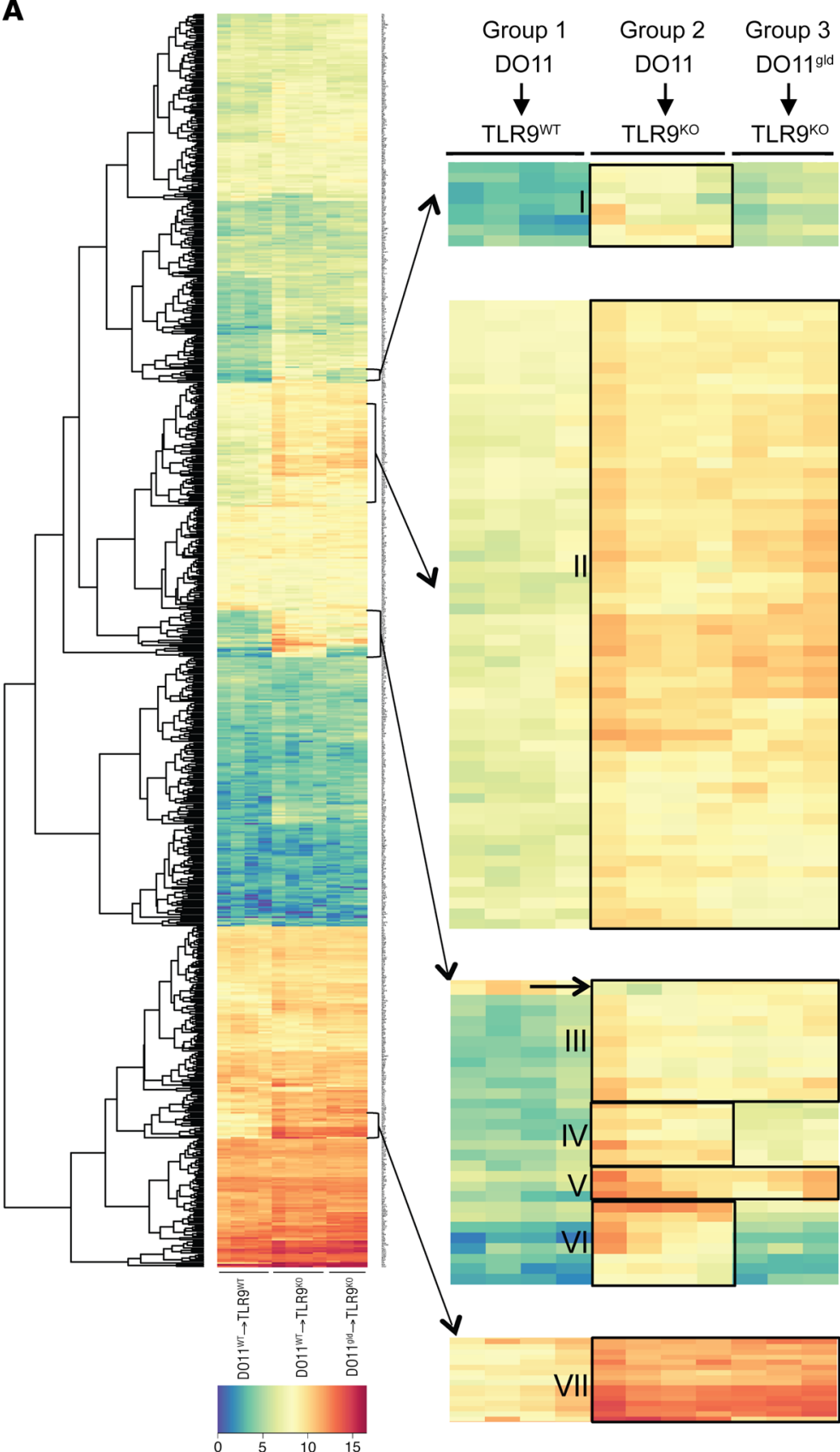

B

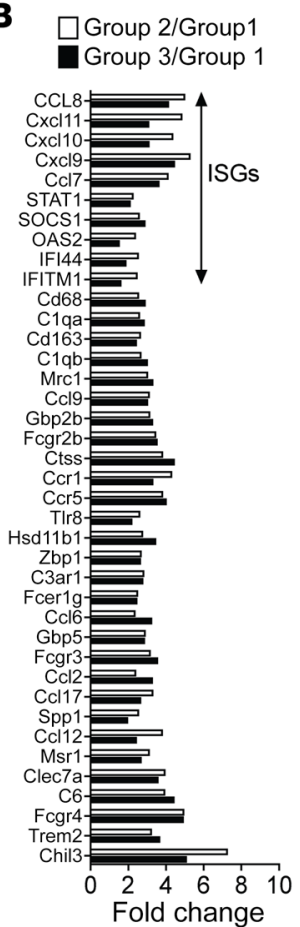

C

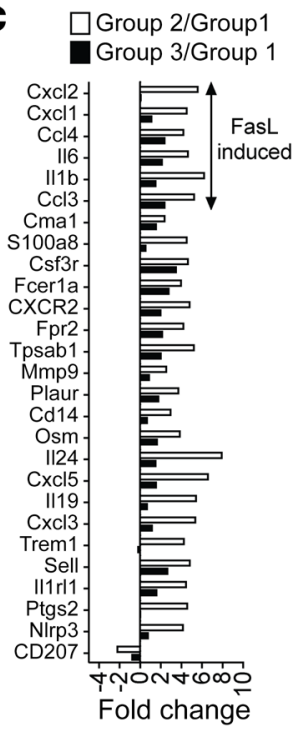

Figure 6. Gene expression in the skin of mice injected with D011 or D011 ${ }^{\text {gld }} \mathbf{T}$ cells. Skin biopsies were obtained from TLRgWT or TLR9 ${ }^{\mathrm{KO}}$ Ii-TCO mice injected with D011 or D011gld T cells. (A) Heatmap showing hierarchical clustering of the 750 genes in the NanoString murine cancer immune code set (left) with data presented as $\log _{2}$-transformed values on a scale of 0 (blue) to 15 (red). Genes above the threshold of mean+2SD of the background were considered for analysis. Enlarged images of gene clusters are designated by arrows (right). Small black arrow denotes the gene downregulated in group 2 compared with group 3. (B) Genes similarly expressed by groups 2 and 3 with a fold change of more than 2.5 relative to group 1 and with $P>0.05$ (not significant) between groups 2 and 3 . (C) Genes differentially expressed by groups 2 and 3 with fold change of more than 2.5 for group 2 relative to group 1 and $P<0.05$ (significant) between groups 2 and 3. Group 1, $n=4$; group 2, $n=4$; and group $3, n=3$. on the expression of TLR7. We have now developed a rapid-onset inducible model of systemic autoimmunity, dependent on the loss of TLR9 and the expression of TLR7, which should facilitate investigation of the regulatory properties of TLR9. These mice resemble other murine models of SLE with regard to splenomegaly, lymphocyte activation, and autoantibody production. Fortuitously, they also develop severe cutaneous inflammation that recapitulates many of the features of human cutaneous lupus.

SLE is considered a type I IFN-driven disease, and SLE patients frequently have a strong IFN signature in peripheral blood mononuclear cells (PBMCs) $(52,53)$. pDCs are considered a major source of type I IFN in these patients (48). Cells isolated from the skin of CLE patients also express an IFN signature attributed to pDCs that traffic to the skin of these patients (50). Type I
IFNs have been implicated in the abnormal activation of a variety of cell types that contribute to SLE pathogenesis, including the generation of IFN- $\gamma$-producing $\mathrm{CD}^{+} \mathrm{T}$ effector subsets (54-56). DO11 $\rightarrow$ TLR9 ${ }^{\mathrm{KO}}$ Ii-TGO mice also showed an IFN signature in the skin that correlated with high numbers of skin-infiltrating DO11 IFN- $\gamma$-producing effector cells. To determine whether type I IFN contributed to the development of skin lesions in our model, we treated DO11 $\rightarrow$ TLR9 ${ }^{\text {KO }}$ Ii-TGO mice with a blocking Ab specific for IFN-aR (46). This same Ab has been used to prevent disease onset in SLE-prone BXSB mice (57). We found that IFN-aR blockade not only prevented the development of skin disease, but also prevented the accumulation of pDCs in the skin. These data are consistent with the notion that pDCs play a pivotal role in our model of LLSI, but also point to an important type I IFN- 
A
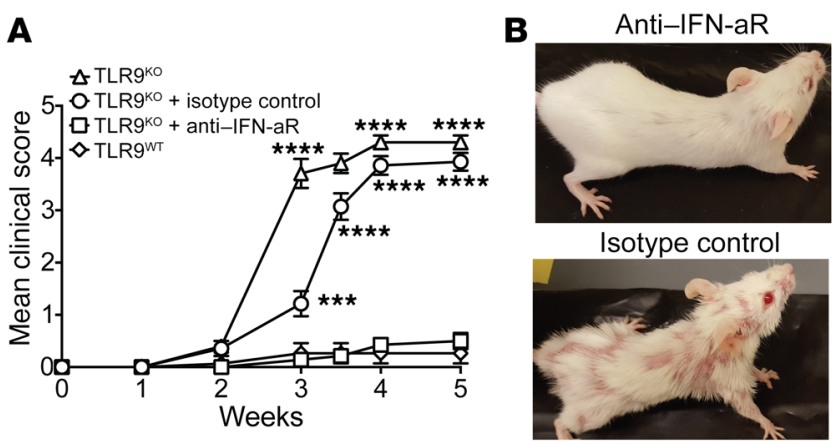

C

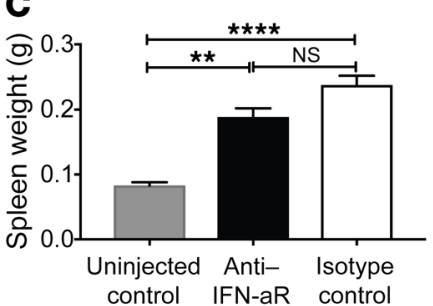

D
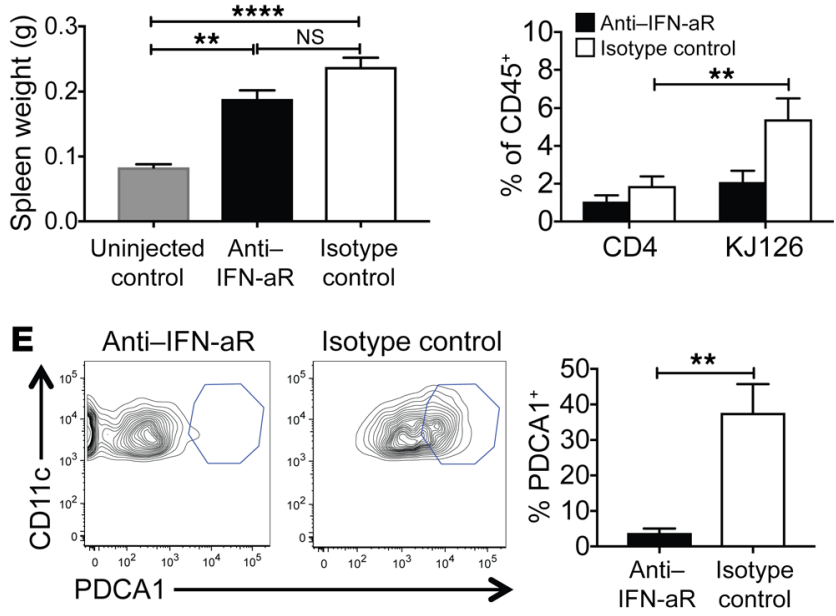

dependent feedback loop in the recruitment and/or maintenance of pDCs in the skin.

Our model depends on a membrane-bound OVA fusion protein, TGO, that incorporates the transmembrane and cytosolic domains of the transferrin receptor and therefore constitutively cycles to the endocytic compartment. Previous studies have shown that DO11 $\times$ TGO mice have a high frequency of Tregs in both the thymus and peripheral lymphoid tissue that is not seen in TGO $^{\text {neg }}$ DO11 mice (28). Therefore, it is highly likely that thymic epithelial cells express low levels of TGO, even in the absence of Dox, and this results in mice that are essentially TGO tolerant. In this context, TGO can be considered a pseudo-autoantigen. This level of tolerance needs to be maintained in order to prevent the development of autoimmune responses. In fact, in order to initiate a response in our Ii-TGO mice, we needed to transfer activated $\mathrm{T}$ cells and use sublethally irradiated Ii-TGO recipients. Sublethal irradiation is thought to create "space" for lymphocyte expansion, but may also lead to the accumulation of cell debris and the production of proinflammatory chemokines that further promote a break in tolerance. In contrast, previous studies by Gratz et al. have shown that the injection of naive DO11 $\mathrm{T}$ cells into Doxtreated K5-TGO mice, where TGO expression is limited to KCs, results in acute inflammation in the skin that then resolves spontaneously with the expansion of peripherally derived Tregs (58). The inability of naive $\mathrm{T}$ cells to induce autoimmunity in Ii-TGO mice suggests that, under homeostatic conditions, TGO expression by MHC class $\mathrm{II}^{+}$cells plays an important role in maintaining a state of tolerance to self-antigens. However, once DO11 $\rightarrow$ Dox $/ 400 R$
Figure 7. Type I IFN blockade abrogates skin disease in TLR9 ${ }^{\mathrm{ko}}$ Ii-TGO mice. (A) Development of skin disease in TLRg ${ }^{\mathrm{KO}}$ mice or $\mathrm{TLRg}^{\mathrm{K} 0}$ mice treated with anti-IFN-aR mAb or isotype control mAb. (B) Representative images at 5 weeks after T cell injection. (C) Spleen weights of experimental and unmanipulated (no T cells or mAb) controls. (D) Percentages of $\mathrm{CD}^{+}$and $\mathrm{CD} 4^{+} \mathrm{KJ} 126^{+}$cells in $\mathrm{CD} 45^{+}$skin cell gate. (E) Representative FACS plots and compiled data showing percentages of CD11c ${ }^{+} P D C A 1^{+} p D C s$ in $\mathrm{CD}_{45}{ }^{+} \mathrm{CD} 11 \mathrm{~b}^{-} \mathrm{CD} 11 \mathrm{c}^{+}$skin cell gate. Data are shown as mean $\pm \mathrm{SEM}$ from 2 independent experiments and are representative of $n=6$ mice per group. ${ }^{* *} P<0.01$; ${ }^{* * *} P<0.001 ;{ }^{* * *} P<0.0001,2$-tailed Student's $t$ test and 2-way ANOVA with Šidák's multiple-comparison test.

TLR9 ${ }^{\mathrm{KO}}$ Ii-TGO mice develop skin lesions, inflammation is not self-resolving, perhaps because the cytokine milieu is so strongly IFN driven that peripheral Treg expansion is prevented. Determining whether DO11 Ii-TGO mice can be "cured" by the transfer of exogenously derived DO11 Treg cells will be an important direction for future studies.

Although we expected TLR9 deficiency to amplify the overall autoimmune response in the Ii-TGO system, we did not anticipate the dramatic impact on the skin, as skin lesions are not routinely observed in other TLR9-deficient SLE-prone strains. KCs are known to express TLR9 (59), upregulate MHC class II in TLR9 ${ }^{\text {KO }}$ Ii-TGO mice, and thereby become a target for skin-infiltrating DO11 effector cells (60). MHC class II expression is a sign of activation, and activated KCs make $\mathrm{T}$ cell chemokines such as CXCL9, CXCL10, and CXCL11 (61), genes that are upregulated in the skin of DO11 $\rightarrow$ TLR9 ${ }^{\text {Ко }}$ Ii-TGO mice (Figure 6). The recruitment of DO11 Th1 cells to the skin can then presumably drive a chronic response by provoking continuous KC death and potentially KC chemokine/cytokine production. KCs also make their own unique form of type I IFN, IFN- $\kappa$, which may also contribute to the strong Th1 phenotype of the infiltrating T cells ( 7 ). Whether TLR9 deficiency in KCs per se contributes to disease amplification will need to be addressed through the analysis of Ii-TGO mice with KC-specific deletion of TLR9.

TLR9 deficiency clearly has a profound effect on the differentiation of DO11 $\mathrm{T}$ cells, leading to the specific expansion of Th1 $\mathrm{T}$ cells in both the skin and sdLNs. Exactly how TLR9 deficiency regulates $\mathrm{T}$ cell fate remains unresolved. It has been proposed that TLR9 and TLR7 compete for binding with Unc93b1 and that the absence of TLR9 results in a stronger TLR7 response that would be more likely to elicit a pathogenic response $(62,63)$. This idea is supported by published studies in immortalized cell lines and BM-derived macrophages, but not in BM-derived DCs or B cells $(64,65)$. It is also possible that TLR9 uniquely activates negative regulators of innate immune responses or contributes to the clearance of cell debris $(66,67)$. It has recently been reported that B cells from SLE patients are hyporesponsive to TLR9, but not TLR7, ligands (68). The role of TLR9 in SLE in additional cell types continues to be an active area of investigation by our lab and others, and the DO11 $\rightarrow \mathrm{Ii}-\mathrm{TGO}$ model is likely to serve as a useful tool for addressing this topic. A better understanding of the cell type(s) directly affected by TLR9 deficiency, under competitive conditions, should enable us to focus attention on the most relevant effector population(s). Published studies implicate TLR9 ${ }^{\mathrm{KO}} \mathrm{B}$ cells, but do not rule out a potential contribution of additional TLR9 ${ }^{\mathrm{KO}}$ APCs or phagocytic cells. 
A

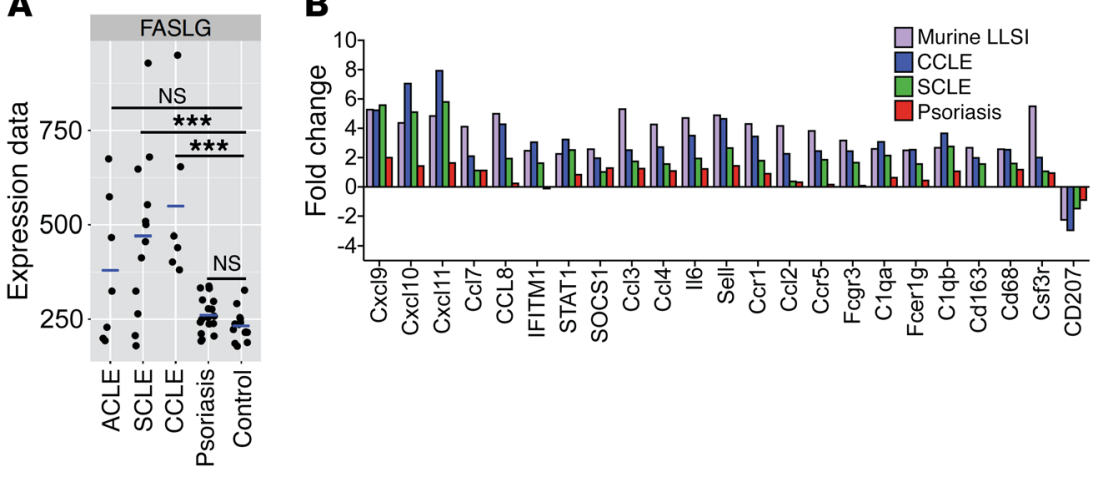

Figure 8. Gene expression in human CLE. (A) Microarray analysis showing FasL transcript levels in healthy control skin. $n=13$. CLE patient skin (ACLE, $n=7$; SCLE, $n=12$; CCLE, $n=6$ ) and psoriasis patient skin $(n=17) .{ }^{* *} P<0.001$. Benjamini-Hochberg procedure was used for multiple comparisons. (B) Fold change of gene expression levels, determined by NanoString analysis, of CCLE, SCLE, and psoriasis lesional skin biopsies relative to healthy control skin biopsies. Genes above background (mean $+2 \mathrm{SD}$ of negative controls) with fold change of 2.0 or above and $P<0.05$ in CCLE relative to healthy controls were selected and compared with genes with fold change greater than 2.5 and $P<0.05$ in D011 $\rightarrow$ TLRg $^{\text {Ko }}$ (group 2) mice relative to control D011 $\rightarrow$ TLRgWT (group 1) li-TCO mice. $n=3$ each of CCLE, SCLE, psoriasis subjects, and healthy controls. Benjamini-Yekutieli procedure was used for multiple comparisons.

Th1 functional analyses routinely focus on IFN- $\gamma$. Certainly, IFN- $\gamma$ plays an important role in macrophage activation during host defense. Moreover, a number of recent studies have identified a critical role for IFN- $\gamma$ in GC responses $(69,70)$, and we consistently detect robust GC responses in our DO11 $\rightarrow$ TLR9 ${ }^{\mathrm{KO}}$ Ii-TGO mice. A substantial subset of Th1 cells also express FasL, and FasL mediates a range of effector functions that can also contribute to lupus pathology, including the induction of apoptosis as well as the production of proinflammatory cytokines $(71,72)$. Nevertheless, the complete absence of skin lesions in TLR9 ${ }^{\mathrm{KO}}$ Ii-TGO recipients of FasL-deficient T cells was not anticipated. KCs express reasonably high levels of Fas, and the large number of potential $\mathrm{KC}$ targets could explain the skin-centric phenotype of Ii-TGO mice. Fas is also upregulated by many of the CD11 $\mathrm{b}^{+}$cells that infiltrate the skin, providing additional targets for $\mathrm{FasL}^{+} \mathrm{T}$ cells. However, the gene expression data strongly suggest that FasL induction of proinflammatory chemokines is also a key factor. Most genes upregulated in the skin of DO11 $\rightarrow \mathrm{TLR}^{\mathrm{KO}}$ versus DO11 $\rightarrow \mathrm{TLR}^{\mathrm{WT}}$ mice were

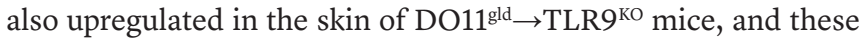
included the majority of IFN-inducible genes. The limited set of genes that distinguished DO11 $\rightarrow$ TLR9 ${ }^{\mathrm{KO}}$ from $\mathrm{DO}^{\mathrm{gld}} \rightarrow \mathrm{TLR}^{\mathrm{KO}}$ skin included many of the myeloid and neutrophil chemokines induced in FasL-stimulated macrophages and other cell types $(43,44)$. Importantly, FasL is upregulated in the skin of CLE, but not psoriasis, patients. Small-molecule FasL inhibitors are under development and have been shown to be highly effective in the blockade of FasL-dependent ocular disorders (73). Related compounds may eventually be appropriate for the treatment of FasLdependent lupus-associated skin disease.

There may also be a connection between FasL and TLR9. In addition to the proinflammatory chemokines listed above, FasL has also been shown to induce the rapid release of HMGB1 through an IRAK4-dependent mechanism (74). HMGB1 has been further shown to bind to DNA and enhance responses to TLR9 ligands through engagement of the receptor for advanced glycation end products (RAGE) and perhaps other receptors $(75,76)$. These studies involved assay systems that measured immunostimulatory effects of DNA/TLR9 engagement. However, as discussed, in the context of murine models of SLE, TLR9 deficiency exacerbates disease outcome. Remarkably, RAGE-deficient B6/lpr mice also develop more severe nephritis (77). Whether FasL-induced HMGB1, under the appropriate conditions, can also contribute to a TLR9-mediated negative feedback loop is yet one more question that can be addressed in future studies. It is well known that both Fas and FasL deficiency can result in systemic autoimmunity with features of SLE, and it has been assumed these models reflect the failure of autoreactive $\mathrm{T}$ cells or $\mathrm{B}$ cells to undergo apoptosis. However, Siegel et al. have recently shown that mice expressing a Fas mutant that is incapable of moving into lipid rafts and transmitting an apoptotic signal do not develop any indications of lymphoproliferative disease or autoimmunity (78). It follows that autoimmunity in Fas and FasL-deficient mice is not due to the failure of FasL to induce apoptosis. Whether FasL can induce the release of HMGB1 from other cells that might negatively regulate autoimmune responses remains to be determined.

In summary, we have developed a murine model that recapitulates many of the features of CLE, including histological changes, a dominant role for Th1 FasL-producing effector cells, a strong IFN signature, production of myeloid chemokines, and the recruitment of pDCs and inflammatory monocytes to the skin. Importantly, this LLSI depends on the transfer of DO11 $\mathrm{T}$ cells into sublethally irradiated recipients. Irradiation may provide an open niche for $\mathrm{T}$ cell expansion, eliminate host OVA-specific Tregs, and/or lead to the release of cell debris. This model is likely to prove useful for mechanistic studies addressing the role of TLR9, TLR7, IFN- $\gamma$, FasL, type I IFNs, and other immune mediators in CLE and for assessing a variety of therapeutic strategies for the treatment of SLE. Small-molecule FasL inhibitors are under development and have been shown to be highly effective in the blockade of FasL-dependent ocular disorders (73). Related compounds may eventually be appropriate for the treatment of FasLdependent lupus-associated skin disease.

\section{Methods}

Mice. BALB/c mice expressing the OVA fusion protein TGO under the control of a TRE have been described previously (28). Mice expressing an Ii-rtTA (31) were provided by C. Benoist (Harvard Medical School, Boston, Massachusetts, USA) and then backcrossed for over 10 generations to a BALB/c background (Jackson Laboratory). The 2 lines were intercrossed to generate the original Ii-TGO line. BALB/c

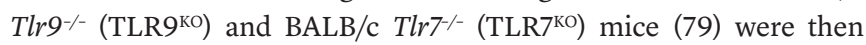
used to generate TLR9 ${ }^{\mathrm{KO}}$ Ii-TGO and TLR7 ${ }^{\mathrm{KO}}$ Ii-TGO mice, which were in turn used to generate the Ii-TGO TLR7/9 double-knockout

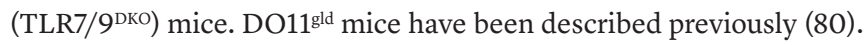


BALB/c DO11 mice (C.Cg-Tg [DO11.10]10 Dlo/J; Jackson Laboratory) and BALB/c IFN- $\gamma^{-/-}$mice (C.129S7 [B6]-Ifng ${ }^{\text {tmlTs }} /$ J; Jackson Laboratory) were used to generate DO11 IFN- $\gamma^{-1-}$ mice.

In vitro culture and adoptive transfer of $T$ cells. Magnetic beadpurified DO11 CD $4^{+} \mathrm{T}$ cells (BD IMag magnetic particles) were activated using OVA peptide-pulsed (323-339, GenScript) irradiated spleen cells (as source of APCs) as described previously (81). $10^{7} \mathrm{~T}$ cells were injected i.v. into sublethally irradiated (4 Gy) age- and sex-matched TLR9 $^{\text {WT }}$, TLR9 ${ }^{\mathrm{KO}}$, TLR7 ${ }^{\mathrm{KO}}$, and TLR7/9 ${ }^{\mathrm{DKO}}$ Ii-TGO recipient mice. To induce expression of the TGO transgene in the MHCII cells, mice were fed with $200 \mathrm{mg} / \mathrm{kg}$ of Dox chow (Bio-Serv).

VPD450-labeled cell-proliferation assay. DO11 $\mathrm{CD}^{+} \mathrm{T}$ cells were labeled with Violet Proliferation Dye (BD Horizon) at a final concentration of $3.5 \mu \mathrm{M} / 1 \times 10^{7}$ cells $/ \mathrm{ml}$ PBS and incubated for 5 minutes in a $37^{\circ} \mathrm{C}$ water bath, followed by quenching with $10 \%$ complete RPMI 1640 medium (cRPMI). $10^{7} \mathrm{VPD}$-labeled T cells were injected i.v. into age- and sex-matched TLR9 ${ }^{\mathrm{WT}}$, TLR9 ${ }^{\mathrm{KO}}$, and TLR7/9 ${ }^{\mathrm{DKO}}$ Ii-TGO recipient mice that were or were not fed with Dox. At day 5 after T cell injection, mice were euthanized and $\mathrm{T}$ cell proliferation in the sdLNs was analyzed by flow cytometry.

Flow cytometry. Single-cell suspensions obtained from spleen, sdLNs, and skin were analyzed by flow cytometry using fluorochromeconjugated mAbs to mouse CD4 (clone RM4-5, Tonbo Biosciences), DO11 TCR (clone KJ126, BD Biosciences), CD45R/B220 (clone RA36B2, Tonbo Biosciences), CD95 (clone Jo2, BD Biosciences), GL-7 (clone GL-7, eBioscience), CD11b (clone M1/70, BioLegend), Ly6C (clone 1A8, BioLegend), Ly6G (clone 1A8, BioLegend), CD11c (clone N418, BioLegend), CD45 (clone 30-F11, eBioscience), CD86 (clone GL1, BioLegend), MHCII (clone I-A/I-E, BioLegend), FasL (clone MFL3, BD Biosciences), CXCR5 (clone SPRCL5, BioLegend), CD279/ PD1 (clone RMP1-30, BioLegend), and CD317/PDCA1 (clone 129c1, BioLegend). Fixable viability dye (ef780, eBiosciences) and Ghost Dye Violet 510 (TONBO Biosciences) were used to distinguish live and dead cells. Intracellular staining was carried out on cells preincubated with PMA (Sigma-Aldrich), ionomycin (Sigma-Aldrich), and GolgiPlug (BD Biosciences) for 4 hours. Cells were permeabilized and fixed with eBioscience FOXP3/transcription factor staining buffer (Invitrogen) and subsequently incubated with fluorochrome-conjugated $\mathrm{mAb}$ to mouse IFN- $\gamma$ (clone XMG1.2, eBioscience), IL17a (clone ebio17B7, eBioscience), Tbet (clone ebio-4B10, eBioscience), GATA3 (clone TWAJ, eBioscience), and ROR $\gamma \mathrm{T}$ (clone B2D, eBioscience). Flow cytometric analysis was carried out using a BD LSR II with FACSDiva software (BD Biosciences), and analysis was conducted with FlowJo software 9.7.6 (TreeStar).

ANAs. ANAs were detected by immunofluorescence staining of HEp-2 antigen substrate slides (MBL Bion). Bound Ab was detected with DyLight 488-coupled goat anti-mouse IgG Ab (Poly4053, BioLegend). Images were captured on a Nikon E600 microscope (NIS Elements imaging software version 4.3) at $\times 200$ magnification.

ELISpot assay. Ab-forming cells were detected with 96-well multiscreen filter plates that were coated overnight at $4^{\circ} \mathrm{C}$ with $5 \mu \mathrm{g} / \mathrm{ml}$ polyclonal goat-anti mouse $\kappa$ (Southern Biotech) and processed as described previously (64).

Histology. Histologic inflammation was assessed in formalin-fixed paraffin-embedded (FFPE) skin biopsy specimens from mice. Sections of $5 \mu \mathrm{m}$ were deparaffinized and stained with H\&E, periodic acid-Schiff stain (PAS) and Alcian blue stain (82). Images were cap- tured on an Olympus BX51 microscope (NIS Elements imaging software version 3.10$)$ at $\times 100, \times 200$, and $\times 400$ magnification.

Immunofluorescence. Skin tissue was snap-frozen in OCT (TissueTek) medium. For Ig staining, $5 \mu \mathrm{m}$ cryostat sections were fixed in cold acetone, blocked with $5 \%$ goat serum in $1 \%$ BSA $/ 0.1 \%$ Tween 20, and incubated with anti-mouse IgG DyLight-488 Ab (Poly4053, BioLegend) for 1 hour. Nuclei were counterstained and mounted with ProLong Gold Antifade with DAPI (Life Technologies). Images were captured on a Zeiss LSM 700 confocal microscope at $\times 200$ magnification. For TUNEL staining, $5 \mu \mathrm{m}$ paraffin-embedded skin sections were processed per the manufacturer's instructions (In Situ Cell Death Detection Kit, TMR red, MilliporeSigma). Nuclei were counterstained and mounted with ProLong Gold Antifade with DAPI (Life Technologies). Images were captured on a Zeiss LSM 700 confocal microscope at $\times 200$ and $\times 400$ magnification.

Cell isolation from skin. Cells were isolated from the epidermis or combined epidermis/dermis as described previously $(30,83)$. Briefly, to isolate cells from the epidermis, shaved torsal skin was harvested, lightly defatted, and incubated in $2 \mathrm{ml}$ Trypsin-EDTA $(0.5 \%, \times 10$, Thermo Fisher) at $37^{\circ} \mathrm{C}$ for 1 hour. Using forceps, epidermal cells were scraped off into a petri dish containing 10\% cRPMI. Cell suspension was filtered through a $100 \mu \mathrm{m}$ filter, washed with cRPMI, and stained for flow cytometry. To isolate cells from combined epidermis and dermis, shaved torsal skin was harvested, minced, and digested for 45 minutes at $37^{\circ} \mathrm{C}$ with $2.0 \mathrm{mg} / \mathrm{ml}$ collagenase XI from Clostridium histolyticum (Sigma-Aldrich), $0.5 \mathrm{mg} / \mathrm{ml}$ hyaluronidase from bovine testes (Sigma-Aldrich), and $0.1 \mathrm{mg} / \mathrm{ml}$ DNAse (Sigma-Aldrich). Single cells were washed with $10 \% \mathrm{cRPMI}$, filtered through a $100 \mu \mathrm{m}$ filter, and stained for flow cytometry or cultured for intracellular cytokine staining as described above.

Gene expression analysis. For mouse NanoString analysis, skin biopsies from mice were harvested and stored in RNAlater (QIAGEN) overnight at $4^{\circ} \mathrm{C}$ and then transferred to $-80^{\circ} \mathrm{C}$ until processed with the RNeasy Mini Kit (QIAGEN) per the manufacturer's instructions. RNA was quantified using a Nanodrop ND-1000 spectrophotometer (Thermo Fisher Scientific). For RNA analysis, 100 ng total RNA was hybridized to a NanoString cancer immune code set of 750 genes according to the manufacturer's protocol (NanoString Technologies Inc.). The gene expression data were normalized to a set of 6 internal positive and 8 internal negative controls and then to 20 housekeeping genes. Values above the threshold (mean $+2 \mathrm{SD}$ of the negative controls) were considered for analysis. Normalized $\log _{2}$-transformed values were analyzed using nSolver analysis software 3.0, and unbiased hierarchical clustering was used to generate the heatmap in the open-source R-based software (heatmap2) at UMMS. Genes significantly upregulated in group 2 and group 3 and with a fold change value of 2.5 or above relative to group 1 were further analyzed and classified into genes with $P>$ 0.05 and genes with $P<0.05$ between groups 2 and 3 . The BenjaminiYekutieli procedure was used to calculate the FDR for analysis.

For human NanoString and microarray analysis, the UMass Memorial (Worcester, Massachusetts, USA) surgical pathology database (https://www.umassmed.edu/pathology/diagnostic/surgicalpathology/) was queried for FFPE biopsies between 1997 and 2011, and each case was reviewed to verify the diagnosis. ACLE, SCLE, CCLE, and psoriasis samples were identified. Control skin was acquired from "dog ears" resulting from excisions in the dermatology clinic. After sample identification, medical records were reviewed to confirm a 
classical clinical presentation as well as to determine the clinical lupus subtype. All samples were deidentified before use in experiments. For gene expression analysis, human skin biopsies were processed as described earlier (51). Briefly, RNA was extracted with the High Pure RNA Paraffin Kit (Roche) and analyzed by Illumina Bioanalyzer. HumanHT-12 v4.0 Whole-Genome DASL HT Assay (Illumina Inc.) was used for microarray gene expression profiling of FFPE human samples according to the manufacturer's instructions. RNA isolation from FFPE sections, quality control, and subsequent Whole-Genome DASL HT assay were performed by the Genomics Core Facility at the Wistar Institute (Philadelpia, Pennsylvania, USA). Microarray data were normalized, transformed, and presented as base 10 values. Multiple comparisons were done using the Benjamini-Hochberg (FDR) procedure. All original microarray data were deposited in the NCBI's Gene Expression Omnibus database (GEO GSE109248).

NanoString gene expression data were processed as above using the NanoString human cancer immune code set of 730 genes. Twenty genes present in the murine cancer immune code set were not present in the human array. As per the manufacturer's recommendations, RNA samples were adjusted to input of approximately 100 ng of RNA fragments greater than 300 nucleotides, which was then hybridized to the cartridge. Further analysis was performed with the nCounter Analysis System. Values above the threshold (mean+2SD of the negative controls) were considered for analysis. Genes with a fold change value of 2.0 or above and $P<0.05$ relative to the healthy controls were compared with the group 2 genes with fold change greater than 2.5 relative to group 1 mice. The Benjamini-Yekutieli procedure was used to calculate the FDR for analysis.

IFN-aR Ab treatment. Anti-IFN-aR (250 $\mu$ g) (MAR1-5A3) (46) or isotype control $\mathrm{Ab}$ was injected i.p. into 400R/Dox TLR9 ${ }^{\mathrm{KO}}$ Ii-TGO mice ( $n=6$ mice per group) twice a week starting on the day of $\mathrm{T}$ cell injection and continuing until mice were euthanized. The murine anti-IFN-aR Ab clone 5A3 and control murine IgG1 Ab clone $1 \mathrm{~A} 7$ were produced and provided by MedImmune. Clinical monitoring for the development of skin lesions was performed until the mice were euthanized for analysis at 5 weeks after T cell transfer. A 4-point clinical scoring scale was used to quantify skin disease based on the clinical parameters of scaling, alopecia, and erythema, which were each given a score of 0-4. Scores for individual clinical parameters were summed for each mouse.

Statistics. Statistical analyses were performed using Prism software version 7.0 (GraphPad). Experiments are reported as mean \pm SEM. Data were analyzed using a 2-tailed Student's $t$ test for comparison between 2 data sets. Multiple comparisons were analyzed by 1-way ANOVA and 2-way ANOVA, followed by Šidák's multiple-comparison post hoc test. Differences were considered significant at a $P$ value of less than 0.05 .
Study approval. The identification and acquisition of FFPE samples from ACLE, SCLE, CCLE, and psoriasis patient skin were approved by the Institutional Review Board at the University of Massachusetts Medical School. Mice were bred and maintained in the Department of Animal Medicine of the University of Massachusetts Medical School in accordance with the regulations of the American Association for the Accreditation of Laboratory Animal Care. All protocols were approved by the Institutional Animal Care and Use Committee.

\section{Author contributions}

PM and AMR designed the studies and wrote the manuscript. PM performed the experiments and analyzed the data. BZ and KT carried out murine skin cell isolation and FACS analysis. MDR directed the FACS analyses of the skin and edited the manuscript. JEH and WCK provided patient skin biopsy RNA samples, analyzed human microarray data, and edited the manuscript. RE provided mouse anti-IFN-aR and isotype control Abs and edited the manuscript. KLB made the original Ii-TGO transgenic line. TYB derived the TLR9 ${ }^{\mathrm{KO}}$ and TLR7 ${ }^{\mathrm{KO}}$ Ii-TGO mice. AD and KD screened patient skin biopsies and characterized mouse skin histology. ZJ and KAF helped analyze and generate the heatmap from the murine NanoString expression data set.

\section{Acknowledgments}

We thank Stephanie Moses for exceptional technical assistance, Debjani Biswas for helping with genotyping mice, the morphology core at UMMS for tissue histology, and Kerstin Nundel for helpful discussions. This work was supported by the Alliance for Lupus Research Lupus Insight Award and NIH grants R01AR066808 (to AMR) and DP2-AR068130 (to MDR).

Address correspondence to: Ann Marshak Rothstein, Department of Medicine/Rheumatology, LRB 309, University of Massachusetts Medical School, 364 Plantation Street, Worcester, Massachusetts 01605, USA. Phone: 508.856.8089; Email: ann.rothstein@ umassmed.edu.

KLB's present address is: Department of Pediatric HematologyOncology, Children's Hospital of Philadelphia, Philadelphia, Pennsylvania, USA.

TYB's present address is: Department of Obstetrics, Gynecology, and Women's Health, University of Minnesota, Minneapolis, Minnesota, USA.

RE's present address is: Viela Bio, Gaithersburg, Maryland, USA.
1. Kirchhof MG, Dutz JP. The immunopathology of cutaneous lupus erythematosus. Rheum Dis Clin North Am. 2014;40(3):455-474.

2. Hejazi EZ, Werth VP. Cutaneous lupus erythematosus: an update on pathogenesis, diagnosis and treatment. Am JClin Dermatol. 2016;17(2):135-146.

3. Achtman JC, Werth VP. Pathophysiology of cutaneous lupus erythematosus. Arthritis Res Ther. 2015;17:182.

4. Stannard JN, Kahlenberg JM. Cutaneous lupus erythematosus: updates on pathogenesis and associations with systemic lupus. Curr Opin Rheumatol. 2016;28(5):453-459.

5. Jabbari A, et al. Dominant Th1 and minimal Th17 skewing in discoid lupus revealed by transcriptomic comparison with psoriasis. J Invest Dermatol. 2014;134(1):87-95.

6. Tsokos GC. Systemic lupus erythematosus. N Engl J Med. 2011; 365(22):2110-2121.

7. Stannard JN, et al. Lupus skin is primed for IL-6 inflammatory responses through a keratinocytemediated autocrine type i interferon loop. JInvest
Dermatol. 2017;137(1):115-122.

8. Furukawa F, Tanaka H, Sekita K, Nakamura T, Horiguchi Y, Hamashima Y. Dermatopathological studies on skin lesions of MRL mice. Arch Dermatol Res. 1984;276(3):186-194.

9. Leadbetter EA, Rifkin IR, Hohlbaum AM, Beaudette BC, Shlomchik MJ, Marshak-Rothstein A. Chromatin-IgG complexes activate B cells by dual engagement of IgM and Toll-like receptors. Nature. 2002;416(6881):603-607.

10. Lau CM, et al. RNA-associated autoantigens acti- 
vate $B$ cells by combined $B$ cell antigen receptor/Toll-like receptor 7 engagement. JExp Med. 2005;202(9):1171-1177.

11. Viglianti GA, Lau CM, Hanley TM, Miko BA, Shlomchik MJ, Marshak-Rothstein A. Activation of autoreactive $\mathrm{B}$ cells by $\mathrm{CpG}$ dsDNA. Immunity. 2003;19(6):837-847.

12. Rawlings DJ, Schwartz MA, Jackson SW, MeyerBahlburg A. Integration of B cell responses through Toll-like receptors and antigen receptors. Nat Rev Immunol. 2012;12(4):282-294.

13. Elkon KB, Wiedeman A. Type I IFN system in the development and manifestations of SLE. Curr Opin Rheumatol. 2012;24(5):499-505.

14. Berland R, et al. Toll-like receptor 7-dependent loss of $\mathrm{B}$ cell tolerance in pathogenic autoantibody knockin mice. Immunity. 2006;25(3):429-440.

15. Christensen SR, Shupe J, Nickerson K, Kashgarian M, Flavell RA, Shlomchik MJ. Toll-like receptor 7 and TLR9 dictate autoantibody specificity and have opposing inflammatory and regulatory roles in a murine model of lupus. Immunity. 2006;25(3):417-428.

16. Ehlers M, Fukuyama H, McGaha TL, Aderem A, Ravetch JV. TLR9/MyD88 signaling is required for class switching to pathogenic IgG2a and $2 \mathrm{~b}$ autoantibodies in SLE. J Exp Med. 2006;203(3):553-561.

17. Groom JR, et al. BAFF and MyD88 signals promote a lupuslike disease independent of $\mathrm{T}$ cells JExp Med. 2007;204(8):1959-1971.

18. Kono DH, et al. Endosomal TLR signaling is required for anti-nucleic acid and rheumatoid factor autoantibodies in lupus. Proc Natl Acad Sci U S A. 2009;106(29):12061-12066.

19. Lee PY, et al. TLR7-dependent and Fc $\gamma R$ independent production of type I interferon in experimental mouse lupus. JExp Med. 2008;205(13):2995-3006.

20. Nickerson KM, et al. TLR9 regulates TLR7- and MyD88-dependent autoantibody production and disease in a murine model of lupus. J Immunol. 2010;184(4):1840-1848.

21. Santiago-Raber ML, et al. Critical role of TLR7 in the acceleration of systemic lupus erythematosus in TLR9-deficient mice. JAutoimmun. 2010;34(4):339-348.

22. Savarese E, et al. Requirement of Toll-like receptor 7 for pristane-induced production of autoantibodies and development of murine lupus nephritis. Arthritis Rheum. 2008;58(4):1107-1115.

23. Silver KL, Crockford TL, Bouriez-Jones T, Milling S, Lambe T, Cornall RJ. MyD88-dependent autoimmune disease in Lyn-deficient mice. Eur $J$ Immunol. 2007;37(10):2734-2743.

24. Christensen SR, Kashgarian M, Alexopoulou L, Flavell RA, Akira S, Shlomchik MJ. Tolllike receptor 9 controls anti-DNA autoantibody production in murine lupus. J Exp Med. 2005;202(2):321-331.

25. Lartigue A, et al. Role of TLR9 in antinucleosome and anti-DNA antibody production in lpr mutation-induced murine lupus. J Immunol. 2006;177(2):1349-1354.

26. Stoehr AD, et al. TLR9 in peritoneal B-1b cells is essential for production of protective selfreactive IgM to control Th17 cells and severe autoimmunity. JImmunol. 2011;187(6):2953-2965
27. Yu P, et al. Toll-like receptor 9-independent aggravation of glomerulonephritis in a novel model of SLE. Int Immunol. 2006;18(8):1211-1219.

28. Rosenblum MD, Gratz IK, Paw JS, Lee K, MarshakRothstein A, Abbas AK. Response to self antigen imprints regulatory memory in tissues. Nature. 2011;480(7378):538-542.

29. Han H, et al. Thymic stromal lymphopoietin (TSLP)-mediated dermal inflammation aggravates experimental asthma. Mucosal Immunol. 2012;5(3):342-351.

30. Gratz IK, et al. Cutting edge: Self-antigen controls the balance between effector and regulatory $\mathrm{T}$ cells in peripheral tissues. JImmunol. 2014;192(4):1351-1355.

31. van Santen H, Benoist C, Mathis D. A cassette vector for high-level reporter expression driven by a hybrid invariant chain promoter in transgenic mice. J Immunol Methods. 2000;245(1-2):133-137.

32. Pisitkun P, Deane JA, Difilippantonio MJ, Tarasenko T, Satterthwaite AB, Bolland S. Autoreactive $\mathrm{B}$ cell responses to RNA-related antigens due to TLR7 gene duplication. Science. 2006;312(5780):1669-1672.

33. Baima B, Sticherling M. Apoptosis in different cutaneous manifestations of lupus erythematosus. Br J Dermatol. 2001;144(5):958-966.

34. Chang LM, et al. Identification and molecular analysis of glycosaminoglycans in cutaneous lupus erythematosus and dermatomyositis. J Histochem Cytochem. 2011;59(3):336-345.

35. Toberer F, et al. Apoptotic signal molecules in skin biopsies of cutaneous lupus erythematosus: analysis using tissue microarray. Exp Dermatol. 2013;22(10):656-659.

36. Furukawa F, et al. Spontaneous autoimmune skin lesions of MRL/n mice: autoimmune disease-prone genetic background in relation to Fas-defect MRL/1pr mice. J Invest Dermatol. 1996;107(1):95-100.

37. Chan OT, Paliwal V, McNiff JM, Park SH, Bendelac A, Shlomchik MJ. Deficiency in beta(2)-microglobulin, but not CD1, accelerates spontaneous lupus skin disease while inhibiting nephritis in MRL-Fas(lpr) nice: an example of disease regulation at the organ level. JImmunol. 2001;167(5):2985-2990.

38. Yang JQ, et al. CD1d deficiency exacerbates inflammatory dermatitis in MRL-lpr/lpr mice. Eur J Immunol. 2004;34(6):1723-1732.

39. Arps DP, Patel RM. Cutaneous hypertrophic lupus erythematosus: a challenging histopathologic diagnosis in the absence of clinical information. Arch Pathol Lab Med. 2013;137(9):1205-1210.

40. Ko CJ, Srivastava B, Braverman I, Antaya RJ, McNiff JM. Hypertrophic lupus erythematosus: the diagnostic utility of CD123 staining. JCutan Pathol. 2011;38(11):889-892.

41. Narang T, Sharma M, Gulati N, Kaur A. Extensive hypertrophic lupus erythematosus: atypical presentation. Indian J Dermatol. 2012;57(6):504

42. Crowson AN, Magro C. The cutaneous pathology of lupus erythematosus: a review. J Cutan Pathol. 2001;28(1):1-23.

43. Hohlbaum AM, Gregory MS, Ju ST, MarshakRothstein A. Fas ligand engagement of resident peritoneal macrophages in vivo induces apoptosis and the production of neutrophil chemotactic factors. JImmunol. 2001;167(11):6217-6224.

44. Brint E, O'Callaghan G, Houston A. Life in the Fas lane: differential outcomes of Fas signaling. Cell Mol Life Sci. 2013;70(21):4085-4099.

45. Nickerson KM, Cullen JL, Kashgarian M, Shlomchik MJ. Exacerbated autoimmunity in the absence of TLR9 in MRL.Fas(lpr) mice depends on Ifnar1. J Immunol. 2013;190(8):3889-3894.

46. Sheehan KC, et al. Blocking monoclonal antibodies specific for mouse IFN- $\alpha / \beta$ receptor subunit 1 (IFNAR-1) from mice immunized by in vivo hydrodynamic transfection. J Interferon Cytokine Res. 2006;26(11):804-819.

47. Delaney TA, et al. Type I IFNs regulate inflammation, vasculopathy, and fibrosis in chronic cutaneous graft-versus-host disease. J Immunol. 2016;197(1):42-50

48. Rönnblom L, Alm GV. A pivotal role for the natural interferon alpha-producing cells (plasmacytoid dendritic cells) in the pathogenesis of lupus. JExp Med.2001;194(12):F59-F63.

49. Zhou Z, et al. Phenotypic and functional alterations of pDCs in lupus-prone mice. Sci Rep. 2016;6:20373.

50. Farkas L, Beiske K, Lund-Johansen F, Brandtzaeg P, Jahnsen FL. Plasmacytoid dendritic cells (natural interferon- $\alpha / \beta$-producing cells) accumulate in cutaneous lupus erythematosus lesions. Am J Pathol. 2001;159(1):237-243.

51. Rashighi M, et al. CXCL10 is critical for the progression and maintenance of depigmentation in a mouse model of vitiligo. Sci Transl Med. 2014;6(223):223ra23.

52. Baechler EC, et al. Interferon-inducible gene expression signature in peripheral blood cells of patients with severe lupus. Proc Natl Acad Sci U S A. 2003;100(5):2610-2615.

53. Bennett L, et al. Interferon and granulopoiesis signatures in systemic lupus erythematosus blood. JExp Med. 2003;197(6):711-723.

54. Jego G, Palucka AK, Blanck JP, Chalouni C, Pascual V, Banchereau J. Plasmacytoid dendritic cells induce plasma cell differentiation through type I interferon and interleukin 6. Immunity. 2003;19(2):225-234.

55. Pascual V, Farkas L, Banchereau J. Systemic lupus erythematosus: all roads lead to type I interferons. Curr Opin Immunol. 2006;18(6):676-682.

56. Wenzel J, et al. Enhanced type I interferon signalling promotes Th1-biased inflammation in cutaneous lupus erythematosus. JPathol. 2005;205(4):435-442.

57. Baccala R, Gonzalez-Quintial R, Schreiber RD, Lawson BR, Kono DH, Theofilopoulos AN. AntiIFN- $\alpha / \beta$ receptor antibody treatment ameliorates disease in lupus-predisposed mice. J Immunol. 2012;189(12):5976-5984.

58. Gratz IK, et al. Cutting edge: memory regulatory T cells require IL-7 and not IL-2 for their maintenance in peripheral tissues. J Immunol. 2013;190(9):4483-4487.

59. Lebre MC, et al. Human keratinocytes express functional Toll-like receptor 3, 4, 5, and 9. JInvest Dermatol. 2007;127(2):331-341.

60. Gaspari AA, Katz SI. Induction and functional characterization of class II MHC (Ia) antigens on murine keratinocytes. JImmunol. 1988;140(9):2956-2963. 
61. Richmond JM, et al. Keratinocyte-derived chemokines orchestrate $\mathrm{T}$-cell positioning in the epidermis during vitiligo and may serve as biomarkers of disease. J Invest Dermatol. 2017;137(2):350-358.

62. Fukui R, et al. Unc93B1 restricts systemic lethal inflammation by orchestrating Toll-like receptor 7 and 9 trafficking. Immunity. 2011;35(1):69-81.

63. Lee BL, et al. UNC93B1 mediates differential trafficking of endosomal TLRs. Elife. 2013;2:e00291.

64. Nündel K, et al. Cell-intrinsic expression of TLR9 in autoreactive B cells constrains BCR/ TLR7-dependent responses. J Immunol. 2015;194(6):2504-2512.

65. Bossaller L, et al. TLR9 deficiency leads to accelerated renal disease and myeloid lineage abnormalities in pristane-induced murine lupus. JImmunol. 2016;197(4):1044-1053.

66. Acharya M, et al. av Integrins combine with LC3 and atg 5 to regulate Toll-like receptor signalling in B cells. Nat Commun. 2016;7:10917.

67. Sindhava VJ, et al. A TLR9-dependent checkpoint governs B cell responses to DNA-containing antigens. J Clin Invest. 2017;127(5):1651-1663.

68. Gies V, et al. Impaired TLR9 responses in B cells from patients with systemic lupus erythematosus. JCI Insight. 2018;3(5):96795.

69. Jackson SW, et al. B cell IFN- $\gamma$ receptor signal- ing promotes autoimmune germinal centers via cell-intrinsic induction of BCL-6. JExp Med. 2016;213(5):733-750.

70. Domeier PP, et al. IFN- $\gamma$ receptor and STAT1 signaling in B cells are central to spontaneous germinal center formation and autoimmunity. JExp Med. 2016;213(5):715-732.

71. Wajant H, Pfizenmaier K, Scheurich P. Nonapoptotic Fas signaling. Cytokine Growth Factor Rev. 2003; 14(1):53-66.

72. Peter ME, Krammer PH. The CD95(APO-1/ Fas) DISC and beyond. Cell Death Differ. 2003;10(1):26-35.

73. Xiao J, Yao J, Jia L, Lin C, Zacks DN. Protective effect of Met12, a small peptide inhibitor of fas, on the retinal pigment epithelium and photoreceptor after sodium iodate injury. Invest Ophthalmol Vis Sci. 2017;58(3):1801-1810.

74. Altemeier WA, Zhu X, Berrington WR, Harlan JM, Liles WC. Fas (CD95) induces macrophage proinflammatory chemokine production via a MyD88-dependent, caspase-independent pathway. J Leukoc Biol. 2007;82(3):721-728.

75. Tian J, et al. Toll-like receptor 9-dependent activation by DNA-containing immune complexes is mediated by HMGB1 and RAGE. Nat Immunol. 2007;8(5):487-496.

76. Sirois CM, et al. RAGE is a nucleic acid receptor that promotes inflammatory responses to DNA.
JExp Med. 2013;210(11):2447-2463.

77. Goury A, et al. Deletion of receptor for advanced glycation end products exacerbates lymphoproliferative syndrome and lupus nephritis in B6-MRL Fas lpr/j mice. J Immunol. 2015;194(8):3612-3622.

78. Cruz AC, et al. Fas/CD95 prevents autoimmunity independently of lipid raft localization and efficient apoptosis induction. Nat Commun. 2016;7:13895.

79. Bossaller L, et al. TLR9 deficiency leads to accelerated renal disease and myeloid lineage abnormalities in pristane-induced murine lupus. JImmunol. 2016;197(4):1044-1053.

80. Saff RR, Spanjaard ES, Hohlbaum AM, MarshakRothstein A. Activation-induced cell death limits effector function of CD4 tumor-specific T cells. JImmunol. 2004;172(11):6598-6606.

81. Randolph DA, Stephens R, Carruthers CJ, Chaplin DD. Cooperation between Th1 and Th2 cells in a murine model of eosinophilic airway inflammation. J Clin Invest. 1999;104(8):1021-1029.

82. Vincent JG, Chan MP. Specificity of dermal mucin in the diagnosis of lupus erythematosus: comparison with other dermatitides and normal skin. J Cutan Pathol. 2015;42(10):722-729.

83. Ali N, et al. Regulatory T cells in skin facilitate epithelial stem cell differentiation. Cell. 2017;169(6):1119-1129.e11. 